\title{
Cascade Descriptor Observers: Application to Understanding Sitting Control of Persons Living With Spinal Cord Injury
}

\author{
Hajer Srihi *, Thierry-Marie Guerra, Anh-Tu Nguyen, Philippe Pudlo and Antoine Dequidt \\ Université Polytechnique Hauts-de-France, CNRS, UMR 8201 LAMIH, Valenciennes, France
}

People with spinal cord injury (SCl) suffer from a drastic reduction in sitting stability which negatively impacts their postural control. Thus, sitting balance becomes one of the most challenging everyday exercises. To better understand the consequences of this pathology, we have to work with high-sized non-linear biomechanical models implying both theoretical and numerical difficulties. The main goal being to recover unmeasured inputs, the observer should have limited or no simplification at all to provide a better estimation quality. A

OPEN ACCESS

Edited by:

Paulo Lopes Dos Santos, University of Porto, Portugal

Reviewed by:

Sunan Huang,

National University of Singapore,

Singapore

Teresa Azevedo Perdicoulis, University of Trás-os-Montes and Alto

Douro, Portugal

*Correspondence: Hajer Srihi

Hajer.srihi@uphf.fr

Specialty section:

This article was submitted to Control and Automation Systems,

a section of the journa

Frontiers in Control Engineering

Received: 15 May 2021 Accepted: 16 September 2021 Published: 05 November 2021

Citation:

Srihi $H$, Guerra T-M, Nguyen A-T, Pudlo $P$ and Dequidt A (2021)

Cascade Descriptor Observers: Application to Understanding Sitting Control of Persons Living With Spinal

Cord Injury.

Front. Control. Eng. 2:710271. doi: 10.3389/fcteg.2021.710271
Proportional Integral-observer (PI-observer) is designed and its convergence is formulated by linear matrix inequalities (LMI) through convex optimization techniques. Using a unique high-sized observer, the LMI constraints problem can quickly reach current solvers limitations regarding the number of unknown parameters required. A way to solve this issue is to design a cascade observer in order to estimate the unmeasurable torques of a human with SCl. This approach consists in decomposing a biomechanical model into interconnected subsystems and to build "local" observers. The relevance of this approach is demonstrated in simulation and with real-time experimental data.

Keywords: spinal cord injury, biomechanical systems, cascade observers, nonlinear model, Takagi-Sugeno formalism, LMI, spinal cord injury

\section{INTRODUCTION}

People with spinal cord injury (SCI) live with a complete or a partial paralysis of their abdominal and lower back muscles. The consequences on sitting control are visible in everyday activities: either reaching, taking objects, or accessing transport. Among the rehabilitation protocols offered to people with SCI, exercises are designed to teach new motor patterns to stabilize the upper part of the trunk, only through movements of the head and upper limbs (Janssen-Potten et al., 1999). Actually, the occupational therapist has no means of objectively following the learning of these new motor patterns, which must nevertheless guarantee a certain stability of the person in a seated position. The authors aim to provide objective data for the occupational therapist, which should enable him to better follow the learning of new motor patterns aimed at stabilizing the upper part of the body through upper limbs in particular. The authors aim also, like other movements such as walking, to provide real-time estimations to the occupational therapist, such as joint torques and their evolution over time. These variables are unmeasurable by nature, except invasive approaches, and this study proposes a way to estimate them using a model approach. This study is a proof-of-concept work to derive non-measurable (unknown) inputs variables from high-sized non-linear models and apply to people living with SCI. The challenge faced is how to derive non-linear observers for high-sized 
systems that preserve at most the initial non-linear model. The main problem is that simplifications, such as partial linearization, will deteriorate the quality of the estimation, especially for the non-measured inputs as they are the "farthest" from the outputs, thus cumulating the imprecisions.

The case study concerns sitting control of persons living with SCI. This study comes from the difficulties to obtain performance results due to complexity and/or limitations of the results obtained previously ${ }^{1}$. First of all, let us recall an important remark: the people with SCI can only mobilize their upper limbs to stabilize the sitting; thus, the main input torque available for a human, the trunk torque, is not functional. Considering the fact that the person cannot activate the muscles below the (complete) lesion and, therefore, no motor torque can be produced at this level to stabilize the upper part of the human body (when needed), people with SCI must, therefore, adopt new strategies to maintain their stability while sitting (Blandeau, 2018). Therefore, understanding how stability is preserved is important but difficult: the torques cannot be measured, the model is highly non-linear, open-loop is unstable, and closed-loop (in the sense of sensorimotor SCI internal control) is very weakly stable. Thus, modeling will also imply building an internal control to stabilize, which is out of the scope of this study, and has been done in Guerra et al. (2018). It implies a very restricted area of stabilization as very tiny disturbances may destabilize the sitting person with SCI. For the observation part, when using a relatively basic mechanical model called H2AT (for Head-Two-Arms-Trunk), non-linear observers expressed as quasi-LPV models were easily derived. "Easily" is interpreted as LMI constraints problems with a reasonable complexity compatible with actual solvers (Blandeau et al., 2018). From this preliminary H2AT model, a more complex model called S3S (Seated-3-Segment) has been built. It is a planar triple-inverted pendulum represented in the sagittal plane (2D) by the trunk, upper arm, and forearm segments. The idea is to go from its actual 2D-S3S to a 3D-S3S form. Nevertheless, in its 2D actual form and taking a global model, the number of states and nonlinearities lead to LMI constraints problems that are already close to the limits of actual solvers, that is, for the brute way-of-doing thousands of constraints and millions of variables (Guerra et al., 2020). Recalling the initial goal of keeping a model the closest possible to reality, using appropriate techniques it is possible to solve the $2 \mathrm{D}-\mathrm{S} 3 \mathrm{~S}$ observation problem with a unique model, but the fact that the optimization problem is close to the limits of the solvers, it is impossible to follow this way-of-doing to get any solution for a 3D-S3S. In order to be able to get feasible performance solutions, this study proposes to decompose the mechanical model under descriptor forms in interconnected systems, from where descriptor non-linear observers of reduced sizes can be derived from local problems (Lendek et al., 2008) (Gripa et al., 2012). It results in cascaded observers design for descriptor mechanical systems. We notice that partitioning approach applied to a non-linear system as well as in the observer design improves the

${ }^{1}$ Blandeau, M., Guerra, T. M., Dequidt, A., Pudlo, P., and Gagnon, D. H. (2021). “A nonlinear biomechanical model for studying sitting control for people living with a spinal cord injury - IEEE T,” in Control Systems Technology. (under review). modularity and reduce complexity of the initial problem which implies a reduction in computational costs (Lendek et al., 2010). The goal is, thus, to apply the methodology on the 2D-S3S model from where results are already available as well as from real-time experiments. We will show that the methodology is perfectly tractable, with formal proof of convergences and results comparable to the global form of observation used in ${ }^{1}$.

The article is organized as follows. After some notations, the second part recalls the 2D-S3S model and quasi-LPV models or socalled Takagi-Sugeno ones. It also gives a first solution to the estimation of the variables with a unique model is provided in continuous as a basis of comparison. The third part proposes a second solution based on decomposition in two interconnected cascaded local models. It includes a global result of convergence for cascaded descriptor models estimation. The fourth part applies this cascade observer way-of-doing to the 2D-S3S model and proposes a solution as a LMI constraints problem to solve. Fifth part proposes the simulation and real-time experiments compared with the global 2D-S3S observer and shows the relevance of the approach.

Notations and Useful Material: the following notations are adopted all along the study. For a given variable, its argument can be omitted and replaced with (.), for general purpose or when there is no ambiguity. With matrices $A_{i}, i \in\{1, \ldots, r\}$ of appropriate dimension, we define a polytopic description in the following form: $A_{h}=\sum_{i=1}^{r} h_{i}(\cdot) A_{i}$, with the nonlinear functions $h_{i}(\cdot) \geq 0$ being continuous and satisfying the convex sum property $\sum_{i=1}^{r} h_{i}(\cdot)=1$. As usual $(*)$ stands for a transpose quantity $Q+$ (*) for $Q+Q^{T}$, and in a matrix (*) represents the transpose of the entry in the symmetric position. At last, $I_{n} \in \mathbb{R}^{n \times n}$ stands for the identity matrix and $0_{n} \in \mathbb{R}^{n \times n}$ for the matrix of null entries.

\section{STATEMENT OF THE PROBLEM}

The 2D-S3S model has been presented in Guerra et al. (2020) and only its equations are recalled therein. The model Figure $\mathbf{1}$ is a variation of the $2 \mathrm{D}$ triple-inverted pendulum represented in the sagittal plane by the trunk, upper arm, and forearm segments (i.e., segments 0,1 , and 2 , respectively) and interconnected by revolute joints at points $\mathrm{T}$ (trunk), S (shoulder), and $\mathrm{E}$ (elbow), whereas the point $\mathrm{H}$ stands for hands. For a segment $i \in\{0,1,2\}, \Gamma_{i}(t)$ stands for the torque input, $G_{i}$ stands for its center of mass (COM), $m_{i}$ for its mass, $l_{i}$ its length, $l_{G_{i}}$ the length from origin to the COM, and $\mathrm{I}_{G_{i}}$ its moment of inertia. Regression rules are previously used to obtain these constants for a typical $80 \mathrm{~kg}$ male (Fang et al., 2017). The relative set points correspond to: $q_{0}=\theta_{0}, q_{1}+\pi=\theta_{1}-\theta_{0}$, and $q_{2}=\theta_{2}-\theta_{1}$. These points correspond to the rest position, and 0 rad corresponding to the hands-up position.

The system of dynamic equations of the S3S model is obtained by deriving the Lagrangian equation $L=E-U$ with $E=\sum_{i=0}^{2} E_{i}$, the kinetic energy, and $U=\sum_{i=0}^{2} U_{i}$, the potential energy, where the kinetic energy (respectively potential) of the system is equal to the

${ }^{1}$ Blandeau, M., Guerra, T. M., Dequidt, A., Pudlo, P., and Gagnon, D. H. (2021). “A nonlinear biomechanical model for studying sitting control for people living with a spinal cord injury - IEEE T," in Control Systems Technology. (under review). 
sum of the energies of each solid. After some manipulations (see, Blandeau, 2018) the Lagrangian of the S3S model can be written as:

$$
\begin{aligned}
L= & \frac{\dot{\theta}_{0}^{2}}{2}\left(\mathrm{I}_{G_{0}}+m_{0} l_{G_{0}}^{2}+m_{1} l_{0}^{2}+m_{2} l_{0}^{2}\right)+\frac{\dot{\theta}_{1}^{2}}{2}\left(\mathrm{I}_{G_{1}}+m_{1} l_{G_{1}}^{2}+m_{2} l_{1}^{2}\right) \\
& +\frac{\dot{\theta}_{2}^{2}}{2}\left(\mathrm{I}_{G_{2}}+m_{2} l_{G_{2}}^{2}\right)-\dot{\theta}_{0} \dot{\theta}_{1}\left(m_{1} l_{0} l_{G_{1}}+m_{2} l_{0} l_{1}\right) \cos \left(q_{1}\right) \\
& +\dot{\theta}_{0} \dot{\theta}_{2} m_{2} l_{0} l_{G_{2}} \cos \left(q_{2}+q_{1}\right)+\dot{\theta}_{1} \dot{\theta}_{2} m_{2} l_{1} l_{G_{2}} \cos \left(q_{2}\right) \\
& -m_{2} g l_{G_{2}} \cos \left(\theta_{2}\right)-\left(m_{0} g l_{G_{0}}+m_{1} g l_{0}+m_{2} g l_{0}\right) \cos \left(\theta_{0}\right) \\
& -\left(m_{1} g l_{G_{1}}+m_{2} g l_{1}\right) \cos \left(\theta_{1}\right) .
\end{aligned}
$$

For introducing simplicity in the expressions, $I_{q 2}=I_{G_{2}}=$ $m_{2} l_{G_{2}}^{2}, I_{q 1}=I_{G_{1}}+I_{G_{2}}+m_{2} l_{G_{2}}^{2}+m_{1} l_{G_{1}}^{2}+m_{2} l_{1}^{2}$ and $I_{q 0}=I_{G_{0}}+$ $I_{G_{1}}+I_{G_{2}}+m_{1} l_{G_{1}}^{2}+m_{2} l_{1}^{2}+m_{2} l_{G_{2}}^{2}+m_{0} l_{G_{0}}^{2}+m_{1} l_{0}^{2}+m_{2} l_{0}^{2}$; the dynamic is described (Khalil and Dombre, 2004) using $\frac{d}{d t}\left(\frac{\partial L}{\partial \dot{q}_{i}}\right)-\frac{\partial L}{\partial q_{i}}=\Gamma_{i}, i \in\{0,1,2\}$. It results in the following:

$$
\begin{aligned}
& \Gamma_{0}=\ddot{q}_{0}\left(I_{q 0}-2\left(m_{1} l_{0} l_{G_{1}}+m_{2} l_{0} l_{1}\right) \cos \left(q_{1}\right)-2 m_{2} l_{0} l_{G_{2}} \cos \left(q_{2}+q_{1}\right)\right. \\
& \left.+2 m_{2} l_{1} l_{G_{2}} \cos \left(q_{2}\right)\right)+\ddot{q}_{1}\left(I_{q 1}-\left(m_{1} l_{0} l_{G_{1}}+m_{2} l_{0} l_{1}\right) \cos \left(q_{1}\right)\right. \\
& \left.-m_{2} l_{0} l_{G_{2}} \cos \left(q_{2}+q_{1}\right)\right)+\ddot{q}_{2}\left(I_{q_{2}}-m_{2} l_{0} l_{G_{2}} \cos \left(q_{2}+q_{1}\right)\right. \\
& \left.+m_{2} l_{1} l_{G_{2}} \cos \left(q_{2}\right)\right)+\dot{q}_{1}^{2}\left(\left(m_{1} l_{0} l_{G_{1}}+m_{2} l_{0} l_{1}\right) \sin \left(q_{1}\right)\right. \\
& \left.+m_{2} l_{0} l_{G_{2}} \sin \left(q_{2}+q_{1}\right)\right)+\dot{q}_{2}^{2}\left(m_{2} l_{0} l_{G_{2}} \sin \left(q_{2}+q_{1}\right)\right. \\
& \left.-m_{2} l_{1} l_{G_{2}} \sin \left(q_{2}\right)\right)+2 \dot{q}_{0} \dot{q}_{1}\left(\left(m_{1} l_{0} l_{G_{1}}+m_{2} l_{0} l_{1}\right) \sin \left(q_{1}\right)\right. \\
& \left.+m_{2} l_{0} l_{G_{2}} \sin \left(q_{2}+q_{1}\right)\right)+2 \dot{q}_{0} \dot{q}_{2}\left(m_{2} l_{0} l_{G_{2}} \sin \left(q_{2}+q_{1}\right)\right. \\
& \left.-m_{2} l_{1} l_{G_{2}} \sin \left(q_{2}\right)\right)+2 \dot{q}_{1} \dot{q}_{2}\left(m_{2} l_{0} l_{G_{2}} \sin \left(q_{2}+q_{1}\right)\right. \\
& \left.-m_{2} l_{1} l_{G_{2}} \sin \left(q_{2}\right)\right)-\left(m_{0} g l_{G_{0}}+m_{1} g l_{0}+m_{2} g l_{0}\right) \sin \left(q_{0}\right) \\
& +\left(m_{1} g l_{G_{1}}+m_{2} g l_{1}\right) \sin \left(q_{1}+q_{0}\right) \\
& +m_{2} g l_{G_{2}} \sin \left(q_{2}+q_{1}+q_{0}\right) \text {, } \\
& \Gamma_{1}=\ddot{q}_{0}\left(I_{q 1}+2 m_{2} l_{1} l_{G_{2}} \cos \left(q_{2}\right)-\left(m_{1} l_{0} l_{G_{1}}+m_{2} l_{0} l_{1}\right) \cos \left(q_{1}\right)\right. \\
& \left.-m_{2} l_{0} l_{G_{2}} \cos \left(q_{2}+q_{1}\right)\right)+\ddot{q}_{1}\left(I_{q_{1}}+2 m_{2} l_{1} l_{G_{2}} \cos \left(q_{2}\right)\right) \\
& +\ddot{q}_{2}\left(I_{q_{2}}+m_{2} l_{1} l_{G_{2}} \cos \left(q_{2}\right)\right)-\dot{q}_{0}^{2}\left(\left(m_{1} l_{0} l_{G_{1}}+m_{2} l_{0} l_{1}\right) \sin \left(q_{1}\right)\right. \\
& \left.+m_{2} l_{0} l_{G_{2}} \sin \left(q_{2}+q_{1}\right)\right)-\dot{q}_{2}^{2} m_{2} l_{1} l_{G_{2}} \sin \left(q_{2}\right) \\
& -2 \dot{q}_{0} \dot{q}_{2} m_{2} l_{1} l_{G_{2}} \sin \left(q_{2}\right)-2 \dot{q}_{1} \dot{q}_{2} m_{2} l_{1} l_{G_{2}} \sin \left(q_{2}\right)+\left(m_{1} g l_{G_{1}}\right. \\
& \left.+m_{2} g l_{1}\right) \sin \left(q_{1}+q_{0}\right)+m_{2} g l_{G_{2}} \sin \left(q_{2}+q_{1}+q_{0}\right) \text {, } \\
& \Gamma_{2}=\ddot{q}_{0}\left(I_{q_{2}}-m_{2} l_{0} l_{G_{2}} \cos \left(q_{2}+q_{1}\right)+m_{2} l_{1} l_{G_{2}} \cos \left(q_{2}\right)\right) \\
& +\ddot{q}_{1}\left(I_{q^{2}}+m_{2} l_{1} l_{G_{2}} \cos \left(q_{2}\right)\right)+\ddot{q}_{2} I_{q 2} \\
& +\dot{q}_{0}^{2}\left(m_{2} l_{1} l_{G_{2}} \sin \left(q_{2}\right)-m_{2} l_{0} l_{G_{2}} \sin \left(q_{2}+q_{1}\right)\right) \\
& +\dot{q}_{1}^{2} m_{2} l_{1} l_{G_{2}} \sin \left(q_{2}\right)+2 \dot{q}_{0} \dot{q}_{1} m_{2} l_{1} l_{G_{2}} \sin \left(q_{2}\right) \\
& +m_{2} g l_{G_{2}} \sin \left(q_{2}+q_{1}+q_{0}\right) \text {. }
\end{aligned}
$$

With a state vector $x(t)=\left[\begin{array}{l}q(t) \\ \dot{q}(t)\end{array}\right]=\left[\begin{array}{llllll}q_{0} & q_{1} & q_{2} & \dot{q_{0}} & \dot{q_{1}} & \dot{q_{2}}\end{array}\right]^{T} \in \mathbb{R}^{6}$ the following non-linear system is obtained from $\mathrm{Eq}(2)$ :

$$
\left\{\begin{array}{l}
E(q(t)) \dot{x}(t)=A(x(t)) x(t)+S(q(t))+B u(t) \\
y(t)=C x(t)
\end{array}\right.
$$

where $y(t)=q(t) \in \mathbb{R}^{3}$ being the output vector, $u(t)=$ $\left[\begin{array}{lll}\Gamma_{0}(t) & \Gamma_{1}(t) & \Gamma_{2}(t)\end{array}\right]^{T}$ the torque inputs vector, $E(q(t))=\left[\begin{array}{cc}I_{3} & 0_{3} \\ 0_{3} & \tilde{E}(q(t))\end{array}\right], \quad A(x(t))=\left[\begin{array}{cc}0_{3} & I_{3} \\ 0_{3} & \tilde{A}(x(t))\end{array}\right], S(q(t))=\left[\begin{array}{c}0_{3 \times 1} \\ \tilde{S}(q(t))\end{array}\right]$, $B=\left[\begin{array}{l}0_{3 \times 3} \\ I_{3}\end{array}\right]$, and $C=\left[\begin{array}{ll}I_{3} & 0_{3}\end{array}\right]$ and the following matrix expressions is obtained:

$$
\begin{aligned}
\tilde{S}(q)= & -\left[\begin{array}{ccc}
-\left(m_{0} g l_{G_{0}}+m_{1} g l_{0}+m_{2} g l_{0}\right) & m_{1} g l_{G_{1}}+m_{2} g l_{1} & m_{2} g l_{G_{2}} \\
0 & m_{1} g l_{G_{1}}+m_{2} g l_{1} & m_{2} g l_{G_{2}} \\
0 & 0 & m_{2} g l_{G_{2}}
\end{array}\right] \\
& {\left[\begin{array}{c}
\sin \left(q_{0}\right) \\
\sin \left(q_{1}+q_{0}\right) \\
\sin \left(q_{2}+q_{1}+q_{0}\right)
\end{array}\right], }
\end{aligned}
$$

$$
\begin{aligned}
& \tilde{E}(q)=\left[e_{i j}\right]_{i, j \in\{1,2,3\}}, \tilde{A}(x)=\left[a_{i j}\right]_{i, j \in\{1,2,3\}}, \\
& e_{11}=I_{q 0}-2\left(m_{1} l_{0} l_{G_{1}}+m_{2} l_{0} l_{1}\right) \cos \left(q_{1}\right)-2 m_{2} l_{0} l_{G_{2}} \cos \left(q_{2}+q_{1}\right) \\
& +2 m_{2} l_{1} l_{G_{2}} \cos \left(q_{2}\right) \\
& e_{12}=I_{q 1}+2 m_{2} l_{1} l_{G_{2}} \cos \left(q_{2}\right)-\left(m_{1} l_{0} l_{G_{1}}+m_{2} l_{0} l_{1}\right) \cos \left(q_{1}\right) \\
& -m_{2} l_{0} l_{G_{2}} \cos \left(q_{2}+q_{1}\right) \text {, } \\
& e_{13}=I_{q^{2}}-m_{2} l_{0} l_{G_{2}} \cos \left(q_{2}+q_{1}\right)+m_{2} l_{1} l_{G_{2}} \cos \left(q_{2}\right) \text {, } \\
& e_{21}=I_{q 1}-\left(m_{1} l_{0} l_{G_{1}}+m_{2} l_{0} l_{1}\right) \cos \left(q_{1}\right)-m_{2} l_{0} l_{G_{2}} \cos \left(q_{2}+q_{1}\right) \\
& +2 m_{2} l_{1} l_{G_{2}} \cos \left(q_{2}\right) \\
& e_{22}=I_{q 1}+2 m_{2} l_{1} l_{G_{2}} \cos \left(q_{2}\right) \text {, } \\
& e_{23}=I_{q 2}+m_{2} l_{1} l_{G_{2}} \cos \left(q_{2}\right) \text {, } \\
& e_{31}=I_{q 2}-m_{2} l_{0} l_{G_{2}} \cos \left(q_{2}+q_{1}\right)+m_{2} l_{1} l_{G_{2}} \cos \left(q_{2}\right) \text {, } \\
& e_{32}=I_{q 2}+m_{2} l_{1} l_{G_{2}} \cos \left(q_{2}\right) \text {, and } e_{33}=I_{q 2} \text {. } \\
& a_{11}=\dot{q}_{2} m_{2} l_{G_{2}}\left(l_{1} \sin \left(q_{2}\right)-l_{0} \sin \left(q_{2}+q_{1}\right)\right)-\dot{q}_{1}\left(\left(m_{1} l_{0} l_{G_{1}}\right.\right. \\
& \left.\left.+m_{2} l_{0} l_{1}\right) \sin \left(q_{1}\right)+m_{2} l_{0} l_{G_{2}} \sin \left(q_{2}+q_{1}\right)\right) \text {, } \\
& a_{12}=\dot{q}_{2} m_{2} l_{G_{2}}\left(l_{1} \sin \left(q_{2}\right)-l_{0} \sin \left(q_{2}+q_{1}\right)\right)-\left(\dot{q}_{1}+\dot{q}_{0}\right)\left(\left(m_{1} l_{0} l_{G_{1}}\right.\right. \\
& \left.\left.+m_{2} l_{0} l_{1}\right) \sin \left(q_{1}\right)+m_{2} l_{0} l_{G_{2}} \sin \left(q_{2}+q_{1}\right)\right) \text {, } \\
& a_{13}=\left(\dot{q}_{0}+\dot{q}_{1}+\dot{q}_{2}\right) m_{2} l_{G_{2}}\left(l_{1} \sin \left(q_{2}\right)-l_{0} \sin \left(q_{2}+q_{1}\right)\right) \text {, } \\
& a_{21}=\dot{q}_{0}\left(\left(m_{1} l_{0} l_{G_{1}}+m_{2} l_{0} l_{1}\right) \sin \left(q_{1}\right)+m_{2} l_{0} l_{G_{2}} \sin \left(q_{2}+q_{1}\right)\right) \\
& +\dot{q}_{2} m_{2} l_{1} l_{G_{2}} \sin \left(q_{2}\right) \text {, } \\
& a_{22}=\dot{q}_{2} m_{2} l_{1} l_{G_{2}} \sin \left(q_{2}\right), a_{23}=\left(\dot{q}_{0}+\dot{q}_{1}+\dot{q}_{2}\right) m_{2} l_{1} l_{G_{2}} \sin \left(q_{2}\right) \text {, } \\
& a_{31}=\dot{q}_{0}\left(m_{2} l_{0} l_{G_{2}} \sin \left(q_{2}+q_{1}\right)+m_{2} l_{1} l_{G_{2}} \sin \left(q_{2}\right)\right) \\
& -\dot{q}_{1} m_{2} l_{1} l_{G_{2}} \sin \left(q_{2}\right) \text {, } \\
& a_{32}=-\left(\dot{q}_{0}+\dot{q}_{1}\right) m_{2} l_{1} l_{G_{2}} \sin \left(q_{2}\right) \text {, and } a_{33}=0 \text {. }
\end{aligned}
$$

Remark 1: The descriptor form is common for mechanical systems; specifically, because it is a natural way to write equations derived from the Euler-Lagrange method (Skelton et al., 1997; Lendek, et al., 2018). For mechanical systems, the matrix $E[q(t)]$ generally ends up regular, as for the S3S model Eq. 3. Therefore, discussions about the singular case, observability and impulse-free modes (Varga, 1995) are not necessary. The way the problem of observation will be solved is using quasi-LPV or so-called T-S models (Tanaka and Wang 2001), and the Lyapunov approach and the solutions are obtained using sets of linear matrix inequalities 
(LMI) (Boyd et al., 1994). Notice that, as $E[q(t)]$ is regular, a classical state space representation can also be straightforwardly derived from Eq. 3. Nevertheless, a key point to reduce complexity (in term of LMI conservatism) is to keep a constant $B$ matrix (Bouarar, et al., 2010). Lastly, using a descriptor formulation such as Eq. 3 has been shown to lower the conservatism of the result by reducing the number of vertices (Lendek, et al., 2018) (Chadli and Darouach, 2012).

Designing an observer in the continuous case for systems such as model Eq. 3 is difficult for two reasons. The first one concerns the fact that the matrix $A[x(t)]$ depends on non-measured variables of $x(t)$. When dealing with a polytopic representation such as T-S models, it resumes in the so-called non-measured premise variables, which is still an open problem in the general case. The second reason is due to the size of the model and the number of non-linearities that may end with LMI constraints problem not being compatible with the actual solvers. The first part presents a solution to model Eq. 3 observation combining ideas from the studies of Guerra et al. (2015), Zemouche et al. (2008), Ichalal and Guerra (2019). Especially, the polytopic description is used the farthest possible in the development.

\section{Global Continuous Proportional Integral-Observer}

As the main goal is to be able to determine the torques that are unknown inputs, several methods can be considered. Nevertheless, we cannot use a classical unknown input observer (UIO) design (Chen et al., 1996) as the rank condition necessary, that is, $\operatorname{rank}(C \times D)=\operatorname{rank}(C)$, is not fulfilled. Therefore, the design of PI-observers comes at hand. From experiments and trials, a double integrator cascade, that is, $\ddot{\Gamma}_{k} \approx 0 k \in\{0,1,2\}$ is used as a good compromise to realize the torque estimations (Blandeau, 2018). Thus, defining a new variable representing the inputs $u=\left[\begin{array}{lll}\Gamma_{0} & \Gamma_{1} & \Gamma_{2}\end{array}\right]^{T}: d=\left[\begin{array}{l}u \\ \dot{u}\end{array}\right]$, the model Eq. 3 can be written in an extended form as:

$$
\begin{aligned}
& \left\{\begin{array}{l}
E^{e}(q) \dot{x}^{e}=A^{e}(x) x^{e}(t)+S^{e}(q), \\
y(t)=C^{e} x^{e}(t)
\end{array}\right. \\
& x^{e}=\left[\begin{array}{l}
x \\
d
\end{array}\right], \dot{d}=\Gamma \times d=\left[\begin{array}{ll}
0_{3} & I_{3} \\
0_{3} & 0_{3}
\end{array}\right] d,
\end{aligned}
$$

where $E^{e}(q)=\left[\begin{array}{cc}E(q) & 0_{6} \\ 0_{6} & I_{6}\end{array}\right], A^{e}(x)=\left[\begin{array}{ccc}A(x) & {[B} & 0_{6 \times 3} \\ 0_{6} & \Gamma\end{array}\right], S^{e}(q)=\left[\begin{array}{c}S(q) \\ 0_{6 \times 1}\end{array}\right]$, and $C^{e}=\left[\begin{array}{ll}C & 0_{3 \times 6}\end{array}\right]$. An observer for (6) can be built using the following:

$$
\left\{\begin{array}{l}
E^{e}(q) \hat{\ddot{x}}^{e}(t)=A^{e}(\hat{x}) \hat{x}^{e}(t)+S^{e}(q)+K(\cdot)(y-\hat{y}) . \\
\hat{y}(t)=C^{e} \hat{x}^{e}(t)
\end{array} .\right.
$$

Matrix $K($.$) will be defined in (19), according to the way of$ getting LMI constraints conditions. Defining the observation error as $e=x^{e}-\hat{x}^{e}$, considering that $E^{e}(q)$ is non-singular its derivative can be directly written using $E^{e}(q) \dot{e}=$ $A^{e}(x) x^{e}(t)-A^{e}(\hat{x}) \hat{x}^{e}(t)-K(\cdot) C^{e} e$, or equivalently as:

$$
E^{e}(q) \dot{e}=\left(A^{e}(\hat{x})-K(\cdot) C^{e}\right) e+\left(A^{e}(x)-A^{e}(\hat{x})\right) x^{e}(t) .
$$

Notice that (8) includes an extra term not depending explicitly on the observation error and introduces non-measurable variables; therefore, asymptotic convergence cannot be guaranteed directly. Next step presents how to derive such asymptotic conditions even in presence of this second term. From the definition of $\tilde{A}(x)=$ $\left[a_{i j}\right]_{i, j \in\{1,2,3\}}$ in (5), let us define the matrix as:

$\Sigma(q)=\left[\begin{array}{ccc}\sigma_{1}+\sigma_{3} & \sigma_{2}-\sigma_{3} & 0 \\ 0 & \sigma_{2} & \sigma_{1}+\sigma_{3} \\ \sigma_{2} & 0 & \sigma_{3}+\sigma_{2}\end{array}\right], \begin{aligned} & \sigma_{1}=\left(m_{1} l_{0} l_{G_{1}}+m_{2} l_{0} l_{1}\right) \sin \left(q_{1}\right) \\ & \sigma_{2}=m_{2} l_{G_{2}} l_{1} \sin \left(q_{2}\right) \\ & \sigma_{3}=m_{2} l_{G_{2}} l_{0} \sin \left(q_{2}+q_{1}\right)\end{aligned}$.

From where we can write the following:

$$
\tilde{A}(x) \dot{q}=\Sigma(q) \Omega(\dot{q}) \dot{q}=\Sigma(q) \Theta(\dot{q}) \dot{q},
$$

where

$$
\Omega(\dot{q})=\left[\begin{array}{ccc}
-2 \dot{q}_{1} & -\dot{q}_{1} & 0 \\
2 \dot{q}_{2} & 2 \dot{q}_{2} & \dot{q}_{2} \\
\dot{q}_{0} & 0 & 0
\end{array}\right] \quad \text { and } \quad \Theta(\dot{q})=
$$

$\left[\begin{array}{ccc}0 & -2 \dot{q}_{0}-\dot{q}_{1} & 0 \\ 0 & 0 & 2 \dot{q}_{0}+2 \dot{q}_{1}+\dot{q}_{2} \\ \dot{q}_{0} & 0 & 0\end{array}\right]$. Of course, $\Omega(\dot{q}) \dot{q}=\Theta(\dot{q}) \dot{q}$

Now it is easy to rewrite the second part of (8) as:

$$
(\tilde{A}(x)-\tilde{A}(\hat{x})) \dot{q}=\Sigma(q)(\Theta(\dot{q})-\Theta(\hat{\dot{q}})) \dot{q} .
$$

Considering
$\left[\begin{array}{ccc}0 & -2 \dot{e}_{0}-\dot{e}_{1} & 0 \\ 0 & 0 & 2 \dot{e}_{0}+2 \dot{e}_{1}+\dot{e}_{2} \\ \dot{e}_{0} & 0 & 0\end{array}\right]\left[\begin{array}{c}\dot{q}_{0} \\ \dot{q}_{1} \\ \dot{q}_{2}\end{array}\right]=\left[\begin{array}{c}-\left(2 \dot{e}_{0}+\dot{e}_{1}\right) \dot{q}_{1} \\ \left(2 \dot{e}_{0}+2 \dot{e}_{1}+\dot{e}_{2}\right) \dot{q}_{2} \\ \dot{e}_{0} \dot{q}_{0}\end{array}\right]$, the following can be written:

$$
(\tilde{A}(x)-\tilde{A}(\hat{x})) \dot{q}=\Sigma(q)\left[\begin{array}{ccc}
-2 \dot{q}_{1} & -\dot{q}_{1} & 0 \\
2 \dot{q}_{2} & 2 \dot{q}_{2} & \dot{q}_{2} \\
\dot{q}_{0} & 0 & 0
\end{array}\right]\left[\begin{array}{c}
\dot{e}_{0} \\
\dot{e}_{1} \\
\dot{e}_{2}
\end{array}\right] .
$$

Now turning back to the extended state:

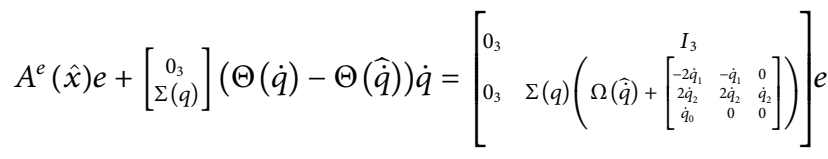

or equivalently:

$$
\begin{aligned}
& A^{e}(\hat{x}) e+\left[\begin{array}{c}
0_{3} \\
\Sigma(q)
\end{array}\right](\Theta(\dot{q})-\Theta(\hat{\dot{q}})) \dot{q}
\end{aligned}
$$

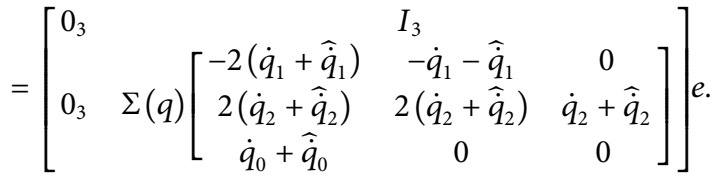

Notice that $\left[\begin{array}{ccc}-2\left(\dot{q}_{1}+\hat{\dot{q}}_{1}\right) & -\dot{q}_{1}-\hat{\dot{q}}_{1} & 0 \\ 2\left(\dot{q}_{2}+\dot{\dot{q}}_{2}\right) & 2\left(\dot{q}_{2}+\dot{\dot{q}}_{2}\right) & \dot{q}_{2}+\hat{\dot{q}}_{2} \\ \dot{q}_{0}+\dot{\dot{q}}_{0} & 0 & 0\end{array}\right]=D(\dot{q}+\hat{\dot{q}}) U$ with $U=$ $\left[\begin{array}{ccc}-2 & -1 & 0 \\ 2 & 2 & 1 \\ 1 & 0 & 0\end{array}\right]$ and $D(\dot{q}+\widehat{\dot{q}})=\operatorname{diag}\left(\left[\begin{array}{lll}\dot{q}_{1}+\hat{\dot{q}}_{1} & \dot{q}_{2}+\hat{\dot{q}}_{2} & \dot{q}_{0}+\hat{\dot{q}}_{0}\end{array}\right]\right)$; therefore, a new writing of (8) is: 


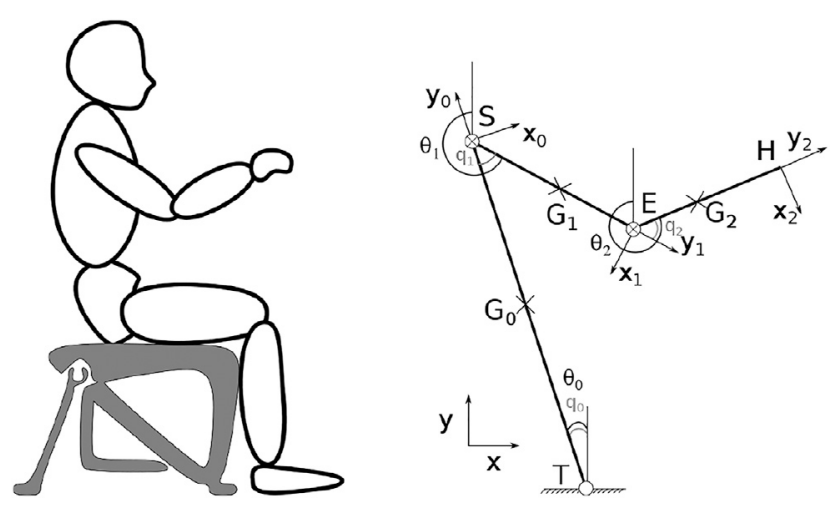

FIGURE 1 | S3S Model, the joint $T$ is free whereas joints $S$ and $E$ are active.

$$
\left[\begin{array}{ccc}
I_{3} & 0_{3} & 0_{3 \times 6} \\
0_{3} & \tilde{E}(q) & 0_{3 \times 6} \\
0_{6 \times 3} & 0_{6 \times 3} & I_{6}
\end{array}\right] \dot{e}=(\underbrace{\left[\begin{array}{cc}
{\left[\begin{array}{cc}
0_{3} & I_{3} \\
0_{3} & \Sigma(q) D(\dot{q}+\hat{q}) U
\end{array}\right]} & \left.\left[\begin{array}{cc}
0_{3} & 0_{3} \\
I_{3} & 0_{3}
\end{array}\right]\right] \\
\Gamma
\end{array}\right]}_{\Phi(\cdot)}-K(\cdot)\left[\begin{array}{ll}
I_{3} & 0_{3 \times 9}
\end{array}\right]) e .
$$

It can be seen that under an assumption of boundedness of the state variables, it is possible from (14) that is strictly equivalent to (8), to derive asymptotic conditions. Let us consider a polytopic form of (14) with four measured variables and three using nonmeasured variables. The four functions $\sin \left(q_{1}\right), \sin \left(q_{2}\right), \cos \left(q_{1}\right)$, and $\cos \left(q_{2}\right)$ are only needed for designing the polytope as $\sin \left(q_{2}+q_{1}\right)$ and $\cos \left(q_{2}+q_{1}\right)$ are derived using classical trigonometric relations. Therefore, $\tilde{E}(q)$ and $\Sigma(q)$ are exactly represented with $2^{4}$ vertices, and with the definition of $D(\dot{q}+\hat{q})$, the three functions $\dot{q}_{0}+\hat{\dot{q}}_{0}, \dot{q}_{1}+\widehat{\dot{q}}_{1}$, and $\dot{q}_{2}+\widehat{\dot{q}}_{2}$ allow to fully represent (14) with $2^{4} \times 2^{3}=128$ vertices. Now, consider the exact polytopic representation of (14) as:

$$
\sum_{i=1}^{16} v_{i}(q) E_{i} \dot{e}=\sum_{i=1}^{16} \sum_{j=1}^{8} v_{i}(q) h_{j}(\dot{q}+\hat{\dot{q}})\left(\Phi_{i j}-K_{i}(\cdot) C\right) e .
$$

For each of the seven non-linearities, a sector non-linearity approach (SNA) is applied (Tanaka \& Wang, 2001). Considering a bounded non-linearity, $z_{j} \in\left[\underline{z}_{j}, \bar{z}_{j}\right]$, the sector is defined as:

$$
z_{j}=\underline{z}_{j} \eta_{0}^{j}\left(z_{j}\right)+\bar{z}_{j} \eta_{1}^{j}\left(z_{j}\right),
$$

where $\eta_{0}^{j}\left(z_{j}\right)=\frac{\bar{z}_{j}-z_{j}}{\bar{z}_{i}-z}$ and $\eta_{1}^{j}\left(z_{j}\right)=1-\eta_{0}^{j}\left(z_{j}\right)$. Each vertex of the polytope will correspond to a product of the weighting functions $\eta_{0}^{j}\left(z_{j}\right): h_{i}(z)=\prod_{j=1}^{r} \eta_{i}^{j}\left(z_{j}\right)$. Of course, the convex property of the weighting functions $\eta_{0}^{j}\left(z_{j}\right)$ remains for $h_{i}(z)$, that is, $h_{i}(z) \geq 0$ and $\sum_{i=1}^{m} h_{i}(z)=1$. For the considered model, the compact set is directly issued from anatomical constraints of the trunk and upper limbs and corresponds to (Blandeau, 2018) the following:

$$
\Omega_{x}=\left\{\begin{array}{ll}
-20^{\circ} \leq q_{1}-q_{1_{e q}} \leq 60^{\circ} & \left\|\dot{q}_{0}\right\| \leq 29^{\circ} / s \\
-10^{\circ} \leq q_{2}-q_{2_{e q}} \leq 45^{\circ}, & \left\|\dot{q}_{1}\right\| \leq 57 \% / s \\
& \left\|\dot{q}_{2}\right\| \leq 57 \% / s
\end{array}\right\} .
$$

Following the study of Guerra et al. (2015), a LMI constraint problem is given. Find $P=\left[\begin{array}{cc}P_{1} & 0 \\ P_{3} & P_{4}\end{array}\right], \quad P_{1}=P_{1}^{T}>0,\left[\begin{array}{l}K_{1 i} \\ K_{2 i}\end{array}\right]$ such that:

$$
\left[\begin{array}{cc}
P_{3}^{T} \Phi_{i j}-K_{1 i} C+(*) & (*) \\
P_{4}^{T} \Phi_{i j}-K_{2 i} C+P_{1}-E_{i}^{T} P_{3} & -P_{4}^{T} E_{i}-E_{i}^{T} P_{4}
\end{array}\right]
$$

and the final observer form is (Guerra et al., 2015) as follows:

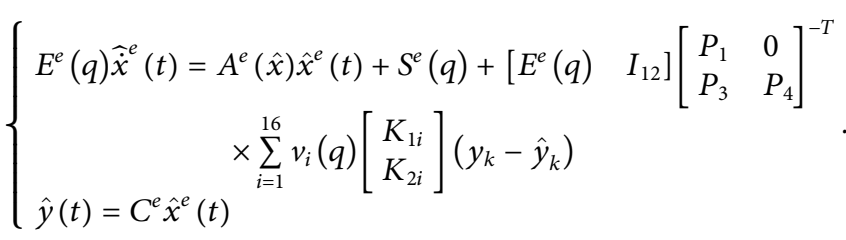

Remark 2: first of all, the observer gain part is only using the four measured non-linearities and functions $v_{i}(q), i \in\{1, \ldots, 16\}$. Second, if (18) holds, then from its last entry $P_{4}^{T} E_{i}+E_{i}^{T} P_{4}>0$, which ensures the regularity of $P_{4}$ and consequently, that $P^{-T}$ needed in (19) is well-defined.

Remark 3: Complexity of problem (18) corresponds to $128+$ 1 LMI constraints, with $n=12$ and $m=3, \frac{n(n+1)}{2}+2 n^{2}+2 n \times m \times$ $2^{4}=1518$ variables. If these figures are reasonable, it is difficult to extend the results considering less conservative LMI problems such as $P_{3}$ and $P_{4}$ depending on the premises, that is, $P_{3}=\sum_{i=1}^{16} \sum_{j=1}^{8} v_{i}(q) h_{j}(\dot{q}+\hat{q}) P_{3 i j}$. In this case, the LMI problem will include depending on the relaxation used, at least 8256 constraints and $\frac{n(n+1)}{2}+2 n^{2} \times 2^{7}+2 n \times m \times 2^{4}=38094$ variables.

From Remark 3, going from 2D-S3S to 3D-S3S looks impossible as the number of non-linearities will increase as well as the number of states. Therefore, solving the problem following a similar approach will only be feasible introducing simplifications. Nevertheless, (19) proposes a solution that will be the basis for comparisons and validation of the next approach.

\section{The Model Decomposed}

This part proposes to solve the problem using a decomposed exact representation of the 2D-S3S model and to show that the reduced problems of observation end with a global proof of convergence with performances comparable to the global PI-observer (19). Thus, this way-of-doing will be compatible with model extensions such as 3D-S3S. To describe in a 


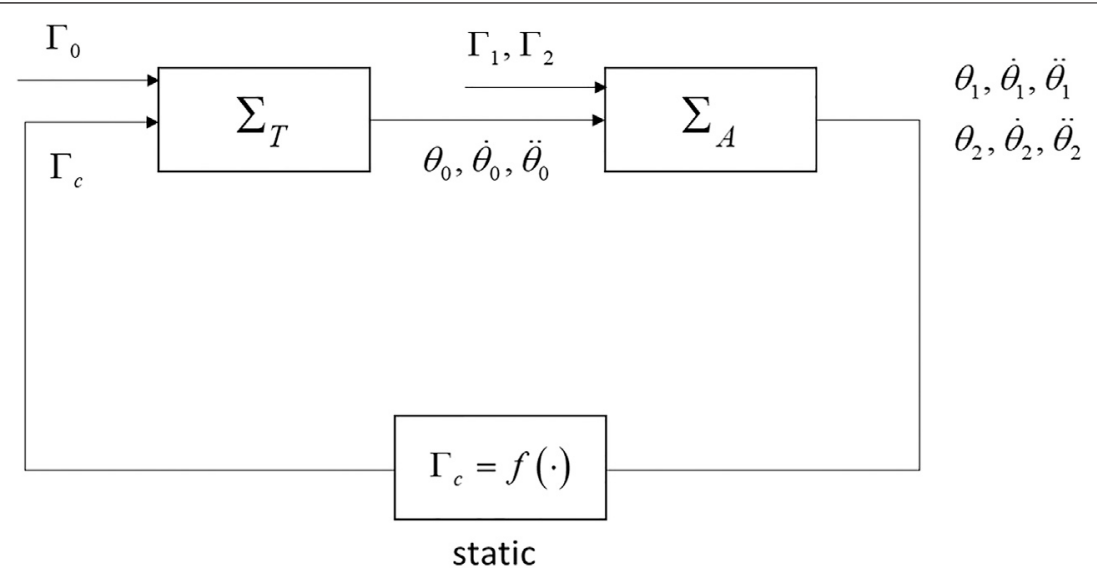

FIGURE 2 | S3S decomposed model $\left(\Sigma_{T}\right.$ and $\left.\Sigma_{A}\right)$.

simpler manner, the models, we introduce the following mechanical parameters:

$$
\begin{aligned}
p_{1} & =I_{G 0}+m_{0} a_{0}^{2}+\left(m_{1}+m_{2}\right) l_{0}^{2}, \quad p_{2}=\left(m_{0} a_{0}+\left(m_{1}+m_{2}\right) l_{0}\right) g, \\
p_{3} & =I_{G 1}+m_{1} a_{1}^{2}+m_{2} l_{1}^{2}, \quad p_{4}=m_{2} a_{2} l_{1}, \quad p_{5}=I_{G 2}+m_{2} a_{2}^{2}, \\
p_{6} & =\left(-m_{1} b_{1}+\left(m_{1}+m_{2}\right) l_{1}\right) g, \\
p_{7} & =m_{2} a_{2} g, \quad p_{8}=m_{1} a_{1} l_{0}+m_{2} l_{0} l_{1}, \quad p_{9}=m_{2} a_{2} l_{0}, \\
p_{10} & =m_{1} a_{1}+m_{2} l_{1} .
\end{aligned}
$$

A subscript " $T$ " stands for trunk and " $A$ " for arm. Therefore, the S3S model is decomposed into, Figure 2, a trunk model $\Sigma_{T}$ (inputs $\Gamma_{0}$ and $\Gamma_{c}$, and outputs $\theta_{0}, \dot{\theta}_{0}$, and $\ddot{\theta}_{0}$ ) that feeds the shoulder + arms model $\Sigma_{A}$ (inputs $\Gamma_{1}, \Gamma_{2}, \theta_{0}, \dot{\theta}_{0}$, and $\ddot{\theta}_{0}$, and outputs $\theta_{i}, \dot{\theta}_{i}$, and $\ddot{\theta}_{i}$, $i \in\{1,2\})$ that provide feedback $\Sigma_{T}$ with a static equation $\Gamma_{c}=f(\cdot)$.

$\Sigma_{T}$, the model of the body trunk, with $\Gamma_{c}$, the coupling torque, corresponds to the following:

$$
p_{1} \ddot{\theta}_{0}=p_{2} \sin \left(\theta_{0}\right)+\Gamma_{c}+\Gamma_{0} .
$$

and in a state space form using the state vector $x_{T}=\left[\begin{array}{c}\theta_{0} \\ \dot{\theta}_{0}\end{array}\right]$ as:

$$
\begin{aligned}
{\left[\begin{array}{cc}
1 & 0 \\
0 & p_{1}
\end{array}\right]\left[\begin{array}{c}
\dot{\theta}_{0} \\
\ddot{\theta}_{0}
\end{array}\right] } & =\left[\begin{array}{cc}
0 & 1 \\
p_{2} \frac{\sin \left(\theta_{0}\right)}{\theta_{0}} & 0
\end{array}\right]\left[\begin{array}{l}
\theta_{0} \\
\dot{\theta}_{0}
\end{array}\right]+\left[\begin{array}{l}
0 \\
1
\end{array}\right]\left(\Gamma_{c}+\Gamma_{0}\right), \\
\theta_{0} & =\left[\begin{array}{ll}
1 & 0
\end{array}\right]\left[\begin{array}{c}
\theta_{0} \\
\dot{\theta}_{0}
\end{array}\right] .
\end{aligned}
$$

The $\Sigma_{A}$ model corresponding to the shoulder and arm equations writes as:

$$
\begin{aligned}
& {\left[\begin{array}{cc}
p_{3} & p_{4} \cos \left(q_{2}\right) \\
p_{4} \cos \left(q_{2}\right) & p_{5}
\end{array}\right]\left[\begin{array}{l}
\ddot{\theta}_{1} \\
\ddot{\theta}_{2}
\end{array}\right]=p_{4} \sin \left(q_{2}\right)\left[\begin{array}{cc}
0 & \dot{\theta}_{2} \\
-\dot{\theta}_{1} & 0
\end{array}\right]\left[\begin{array}{c}
\dot{\theta}_{1} \\
\dot{\theta}_{2}
\end{array}\right]} \\
& +\left[\begin{array}{c}
p_{6} \sin \left(\theta_{1}\right) \\
p_{7} \sin \left(\theta_{2}\right)
\end{array}\right]+\left[\begin{array}{cc}
1 & -1 \\
0 & 1
\end{array}\right]\left[\begin{array}{c}
\Gamma_{1} \\
\Gamma_{2}
\end{array}\right] \\
& +\left[\begin{array}{cc}
p_{8} \sin \left(q_{1}\right) & p_{8} \cos \left(q_{1}\right) \\
p_{9} \sin \left(q_{1}+q_{2}\right) & p_{9} \cos \left(q_{1}+q_{2}\right)
\end{array}\right]\left[\begin{array}{c}
\dot{\theta}_{0}^{2} \\
\ddot{\theta}_{0}
\end{array}\right]
\end{aligned}
$$

From where a state representation is:

$$
\begin{aligned}
& {\left[\begin{array}{cccc}
1 & 0 & 0 & 0 \\
0 & 1 & 0 & 0 \\
0 & 0 & p_{3} & p_{4} \cos \left(q_{2}\right) \\
0 & 0 & p_{4} \cos \left(q_{2}\right) & p_{5}
\end{array}\right]\left[\begin{array}{l}
\dot{\theta}_{1} \\
\dot{\theta}_{2} \\
\ddot{\theta}_{1} \\
\ddot{\theta}_{2}
\end{array}\right]} \\
& =\left[\begin{array}{cccc}
0 & 0 & 1 & 0 \\
0 & 0 & 0 & 1 \\
0 & 0 & 0 & p_{4} \sin \left(q_{2}\right) \dot{\theta}_{2} \\
0 & 0 & -p_{4} \sin \left(q_{2}\right) \dot{\theta}_{1} & 0
\end{array}\right]\left[\begin{array}{c}
\theta_{1} \\
\theta_{2} \\
\dot{\theta}_{1} \\
\dot{\theta}_{2}
\end{array}\right] \\
& +\left[\begin{array}{cc}
0 & 0 \\
0 & 0 \\
1 & -1 \\
0 & 1
\end{array}\right]\left[\begin{array}{c}
\Gamma_{1} \\
\Gamma_{2}
\end{array}\right]+\left[\begin{array}{c}
0 \\
p_{6} \sin \left(\theta_{1}\right) \\
p_{7} \sin \left(\theta_{2}\right)
\end{array}\right] \\
& +\left[\begin{array}{c}
0 \\
0 \\
p_{8} \sin \left(q_{1}\right) \\
p_{9} \sin \left(q_{1}+q_{2}\right) \\
p_{9} \cos \left(q_{1}+q_{2}\right)
\end{array}\right]\left[\begin{array}{l}
\dot{\theta}_{0}^{2} \\
\ddot{\theta}_{0}
\end{array}\right],
\end{aligned}
$$

and considering $\theta_{A}=\left[\begin{array}{l}\theta_{1} \\ \theta_{2}\end{array}\right]$ which represents the measured variables and $x_{A}=\left[\theta_{\dot{\theta}_{A}}^{\theta_{A}}\right]$, the full state including the nonmeasured speeds as:

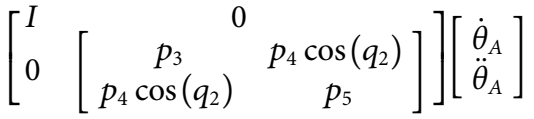

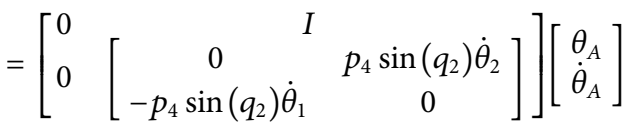

$$
\begin{aligned}
& +\left[\left[\begin{array}{cc}
1 & -1 \\
0 & 1
\end{array}\right]\right]\left[\begin{array}{l}
\Gamma_{1} \\
\Gamma_{2}
\end{array}\right]+\left[\begin{array}{c}
0 \\
D_{a}\left(\theta_{A}\right)
\end{array}\right]+\left[\begin{array}{c}
0 \\
C\left(\theta_{A}\right)
\end{array}\right]\left[\begin{array}{l}
\dot{\theta}_{0}^{2} \\
\ddot{\theta}_{0}
\end{array}\right],
\end{aligned}
$$

where $\quad D_{a}\left(\theta_{A}\right)=\left[\begin{array}{l}p_{6} \sin \left(\theta_{1}\right) \\ p_{7} \sin \left(\theta_{2}\right)\end{array}\right]$ and $C\left(\theta_{A}\right)=$ $\left[\begin{array}{cc}p_{8} \sin \left(q_{1}\right) & p_{8} \cos \left(q_{1}\right) \\ p_{9} \sin \left(q_{1}+q_{2}\right) & p_{9} \cos \left(q_{1}+q_{2}\right)\end{array}\right]$.

Finally, the static coupling term, Figure 2, that feedbacks from $\Sigma_{A}$ to $\Sigma_{T}$ corresponds to the following: 
$\Gamma_{c}=\left[\begin{array}{llll}-p_{10} \sin \left(q_{1}\right) \dot{\theta}_{1} & -p_{9} \sin \left(q_{1}+q_{2}\right) \dot{\theta}_{2} & p_{10} \cos \left(q_{1}\right) & p_{9} \cos \left(q_{1}+q_{2}\right)\end{array}\right]$

$$
\left[\begin{array}{c}
\dot{\theta}_{1} \\
\dot{\theta}_{2} \\
\ddot{\theta}_{1} \\
\ddot{\theta}_{2}
\end{array}\right]-\Gamma_{1}
$$

Thus, $\Sigma_{A}$ equation 21, $\Sigma_{T}$ (24) and the coupling term (25) exactly represent the S3S model (3).

\section{Unknown Input Observation Problem}

In both cases, as previously done for the S3S model (6), a double integrator cascade, that is, $\ddot{\Gamma}_{k} \approx 0 k \in\{0,1,2\}$ is used as a good compromise to realize the torque estimations. For the system $\Sigma_{T}$, the state is augmented to include the torque $\Gamma_{U}=\Gamma_{c}+\Gamma_{0}$. Effectively, at this first stage, there is no way to make a difference between the torques $\Gamma_{c}$ and $\Gamma_{0}$ as they enter at the same place in (20). Therefore, (21) is rewritten in the PI-extended form as:

$$
\begin{aligned}
& {\left[\begin{array}{cc}
{\left[\begin{array}{cc}
1 & 0 \\
0 & p_{1}
\end{array}\right]} & 0 \\
0 & I
\end{array}\right]\left[\begin{array}{l}
\dot{\theta}_{0} \\
\ddot{\theta}_{0} \\
\dot{\Omega}_{T}
\end{array}\right]=\left[\begin{array}{cc}
0 & 1 \\
p_{2} \frac{\sin \left(\theta_{0}\right)}{\theta_{0}} & 0 \\
0 &
\end{array}\right]\left[\begin{array}{cc}
B & 0
\end{array}\right]} \\
& {\left[\begin{array}{c}
\theta_{0} \\
\dot{\theta}_{0} \\
\Omega_{T}
\end{array}\right], \Omega_{T}=\left[\begin{array}{c}
\Gamma_{U} \\
\dot{\Gamma}_{U}
\end{array}\right], J=\left[\begin{array}{ll}
0 & 1 \\
0 & 0
\end{array}\right] .}
\end{aligned}
$$

For the model $\Sigma_{A}(\mathbf{2 4})$, the state is augmented to include the torques $\Gamma_{1}$ and $\Gamma_{2}$. For sake of simplification, we define

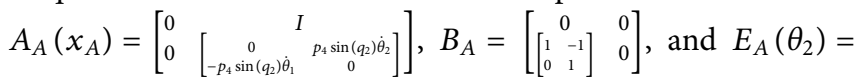

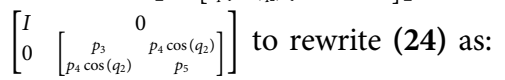

$$
E_{A}\left(\theta_{A}\right) \dot{x}_{A}=A_{A}\left(x_{A}\right) x_{A}+\left[\begin{array}{l}
0 \\
B
\end{array}\right]\left[\begin{array}{l}
\Gamma_{1} \\
\Gamma_{2}
\end{array}\right]+\left[\begin{array}{c}
0 \\
D_{a}\left(\theta_{A}\right)
\end{array}\right]+\left[\begin{array}{c}
0 \\
C\left(\theta_{A}\right)
\end{array}\right]\left[\begin{array}{l}
\dot{\theta}_{0}^{2} \\
\ddot{\theta}_{0}
\end{array}\right],
$$

and its extended PI-extended form with $J_{2}=\left[\begin{array}{cc}0 & I_{2} \\ 0 & 0\end{array}\right]$ and $\Omega_{A}=$ $\left[\begin{array}{llll}\Gamma_{1} & \dot{\Gamma}_{1} & \Gamma_{2} & \dot{\Gamma}_{2}\end{array}\right]^{T}$ as:

$$
\left\{\begin{array}{c}
{\left[\begin{array}{cc}
E_{A}\left(\theta_{2}\right) & 0 \\
0 & I
\end{array}\right]\left[\begin{array}{l}
\dot{x}_{A} \\
\dot{\Omega}_{A}
\end{array}\right]=\left[\begin{array}{cc}
A_{A}\left(x_{A}\right) & B_{A} \\
0 & J_{2}
\end{array}\right]\left[\begin{array}{l}
x_{A} \\
\Omega_{A}
\end{array}\right]} \\
+\left[\begin{array}{c}
0 \\
D_{a}\left(\theta_{A}\right) \\
0
\end{array}\right]+\left[\begin{array}{c}
0 \\
C\left(\theta_{A}\right) \\
0
\end{array}\right]\left[\begin{array}{c}
\dot{\theta}_{0}^{2} \\
\ddot{\theta}_{0}
\end{array}\right] \\
y_{A}=\left[\begin{array}{ll}
I_{2} & 0
\end{array}\right]\left[\begin{array}{l}
x_{A} \\
\Omega_{A}
\end{array}\right]=\theta_{A}=\left[\begin{array}{c}
\theta_{1} \\
\theta_{2}
\end{array}\right]
\end{array} .\right.
$$

Thus, with (26), (28), and the coupling term (25), the goal is to build a cascade of two observers for $\Sigma_{T}$ and $\Sigma_{A}$, and to make their synthesis in order to guarantee an asymptotic convergence as well as performances comparable to a global observer. Before solving the problem for the S3S model, let us give a general result concerning cascade observers in the descriptor form.

\section{CASCADE OBSERVATION PROBLEM}

\section{Conditions for a Separation Principle}

Cascade observation has been studied for interconnected nonlinear and linear systems, for example in Lendek et al. (2008), Gripa et al. (2012). The idea is to build observers independently, in a way that the global performances are satisfied. Thus, we can combine different types of observer regarding the local subsystem concerned. A separation principle is proposed based on a vector comparison principle, and the proof follows similar paths than the observer/control separation principle for quasi-LPV systems (Ma et al., 1998). The advantage of this methodology of estimation is that separate observers can be built from a local subsystem which makes their adjustment less difficult (Lendek, et al., 2010).

Consider the following proposition.

Theorem 1: consider two descriptor systems $\left[E_{i}(\cdot), A_{i}(\cdot), C_{i}(\cdot)\right], i \in\{1,2\}\left(E_{i}(\cdot)\right.$ being always regular) such that it exists in matrices as $K_{i}(\cdot), i \in\{1,2\}$ ensuring that the estimation error systems defined by $E_{i}(\cdot) \dot{e}_{i}=\left[A_{i}(\cdot)-\right.$ $\left.K_{i}(\cdot) C_{i}(\cdot)\right] e_{i}$ are globally asymptotically stable (GAS); knowing it exists as two Lyapunov functions $V_{i}\left(e_{i}\right)$ and scalars $\bar{\lambda}_{i} \geq \underline{\lambda}_{i}>0$, $\mu_{i}>0, \delta_{i}>0$ satisfying the following:

i. $\quad 0<\underline{\lambda}_{i}\left\|e_{i}\right\|^{2} \leq V_{i}\left(e_{i}\right) \leq \bar{\lambda}_{i}\left\|e_{i}\right\|^{2}$.

ii. $\quad \frac{\partial V_{i}\left(e_{i}\right)}{\partial e_{i}} \dot{e}_{i}=\frac{\partial V_{i}\left(e_{i}\right)}{\partial e_{i}} E_{i}^{-1}(\cdot)\left(A_{i}(\cdot)-K_{i}(\cdot) C_{i}(\cdot)\right) \leq-\mu_{i}\left\|e_{i}\right\|^{2}$.

iii. $\left\|\frac{\partial V_{i}\left(e_{i}\right)}{\partial e_{i}}\right\| \leq \delta_{i}\left\|e_{i}\right\|^{2}$.

Consider the System

$$
\begin{aligned}
& {\left[\begin{array}{cc}
E_{1}(\cdot) & 0 \\
E_{12}(\cdot) & E_{2}(\cdot)
\end{array}\right] \dot{e}} \\
& =\left[\begin{array}{cc}
A_{1}(\cdot)-K_{1}(\cdot) C_{1}(\cdot) & 0 \\
A_{12}(\cdot) & A_{2}(\cdot)-K_{2}(\cdot) C_{2}(\cdot)
\end{array}\right] e \text {, }
\end{aligned}
$$

where $E_{12}(\cdot)$ and $A_{12}(\cdot)$ norm-bounded; then (32) is also globally asymptotically stable.

Proof: $E_{i}(\cdot)$ being regular, it is direct that $\left[\begin{array}{cc}E_{1}(\cdot) & 0 \\ E_{12}(\cdot) & E_{2}(\cdot)\end{array}\right]^{-1}=\left[\begin{array}{cc}E_{1}^{-1}(\cdot) & 0 \\ -E_{2}^{-1}(\cdot) E_{12}(\cdot) E_{1}^{-1}(\cdot) & E_{2}^{-1}(\cdot)\end{array}\right] ; \quad$ let $\quad$ us rewrite $A_{i}^{*}(\cdot)=E_{i}^{-1}(\cdot)\left(A_{i}(\cdot)-K_{i}(\cdot) C_{i}(\cdot)\right)$, thus (32) is equivalent to the following:

$$
\dot{e}=\left[\begin{array}{cc}
A_{1}^{*}(\cdot) & 0 \\
E_{2}^{-1}(\cdot)\left(A_{12}(\cdot)-E_{12}(\cdot) A_{1}^{*}(\cdot)\right) & A_{2}^{*}(\cdot)
\end{array}\right] e .
$$

Consider now a positive scalar $\gamma>0$ and the Lyapunov function as:

$$
V\left(e_{1}, e_{2}\right)=V_{1}\left(e_{1}\right)+\gamma V_{2}\left(e_{2}\right)
$$

Its derivative along the trajectories of (33) is: 


$$
\begin{aligned}
\dot{V}\left(e_{1}, e_{2}\right)= & \frac{\partial V_{1}\left(e_{1}\right)}{\partial e_{1}} \dot{e}_{1}+\gamma \frac{\partial V_{2}\left(e_{2}\right)}{\partial e_{2}} \dot{e}_{2} \\
= & \frac{\partial V_{1}\left(e_{1}\right)}{\partial e_{1}} A_{1}^{*}(\cdot) e_{1}+\gamma \frac{\partial V_{2}\left(e_{2}\right)}{\partial e_{2}} A_{2}^{*}(\cdot) e_{2} \\
& +\gamma \frac{\partial V_{2}\left(e_{2}\right)}{\partial e_{2}} E_{2}^{-1}(\cdot)\left(A_{12}(\cdot)-E_{12}(\cdot) A_{1}^{*}(\cdot)\right) e_{1} .
\end{aligned}
$$

Using (30) and Passing at Norms, we get the following:

$$
\begin{aligned}
\dot{V}\left(e_{1}, e_{2}\right) \leq & -\mu_{1}\left\|e_{1}\right\|^{2}-\gamma \mu_{2}\left\|e_{2}\right\|^{2}+\gamma\left\|\frac{\partial V_{2}\left(e_{2}\right)}{\partial e_{2}}\right\| \\
& \times\left\|E_{2}^{-1}(\cdot)\left(A_{12}(\cdot)-E_{12}(\cdot) A_{1}^{*}(\cdot)\right)\right\|\left\|e_{1}\right\|,
\end{aligned}
$$

where $A_{1}^{*}(\cdot)$ is GAS, and $E_{12}(\cdot)$ and $A_{12}(\cdot)$ are norm-bounded; we can write that it exists a positive scalar $M>0$ such that $\left\|E_{2}^{-1}(\cdot)\left(A_{12}(\cdot)-E_{12}(\cdot) A_{1}^{*}(\cdot)\right)\right\| \leq M$. Using condition (31), the bound (36) of the derivative $\dot{V}\left(e_{1}, e_{2}\right)$ holds if:

$$
\dot{V}\left(e_{1}, e_{2}\right) \leq-\mu_{1}\left\|e_{1}\right\|^{2}-\gamma \mu_{2}\left\|e_{2}\right\|^{2}+\gamma \delta_{2} M\left\|e_{1}\right\|\left\|e_{2}\right\| .
$$

Using a completion of square, (37) is equivalent to:

$\dot{V}\left(e_{1}, e_{2}\right) \leq-\left(\mu_{1}-\gamma \frac{\delta_{2}^{2} M^{2}}{4 \mu_{2}}\right)\left\|e_{1}\right\|^{2}-\gamma\left(\sqrt{\mu_{2}}\left\|e_{2}\right\|-\frac{\delta_{2} M}{2 \sqrt{\mu_{2}}}\left\|e_{1}\right\|\right)^{2}$.

As $\gamma>0$ is a free parameter, it can always be set such as $\gamma<\frac{4 \mu_{1} \mu_{2}}{\delta_{2}^{2} M^{2}}$ and thus, $\dot{V}\left(e_{1}, e_{2}\right)<0 \forall\left(e_{1}, e_{2}\right) \neq 0$ which ends the proof.

Remark 4: This result is readily extended to a $n$-cascade of observers that keep the triangular structure of (32) and the normbounded property on its non-diagonal entries. This will be particularly interesting for the future studies considering a 3DS3S model.

From this general result, next part shows how to exploit it to derive the 2D-S3S cascaded observers via LMI constraints problems.

\section{Cascade Proportional Integral-Observers for the S3S Model}

First of all, let us describe the two observers cascade for the S3S case, Figure 3. Recall that only the angles are measured. Thus the first observer of $\Sigma_{T}$ (so-called $\hat{\Sigma}_{T}$, Figure 3) has $\theta_{0}$ and $\hat{\theta}_{0}$ as inputs, and $\hat{\Gamma}_{U}=\hat{\Gamma}_{c}+\hat{\Gamma}_{0}$ as output. The second observer for $\Sigma_{A}$ (so-called $\hat{\Sigma}_{A}$, Figure 3 ) uses the first observer outputs and the angles $\theta_{1}$ and $\theta_{2}$ as inputs. Its outputs are $\hat{\theta}_{i}, \hat{\hat{\theta}}_{i}$, and $\hat{\Gamma}_{i} i \in\{1,2\}$.

We considered the extended body trunk model $\Sigma_{T}$ (26) and transform it in a classical non-descriptor form with: $x_{T}=$ $\left[\begin{array}{l}\theta_{0} \\ \dot{\theta}_{0}\end{array}\right], A_{T}\left(\theta_{0}\right)=\left[\begin{array}{cc}1 & 0 \\ 0 & p_{1}^{-1}\end{array}\right]\left[\begin{array}{cc}0 & 1 \\ p_{2} \frac{\sin \left(\theta_{0}\right)}{\theta_{0}} & 0\end{array}\right], \quad B=\left[\begin{array}{l}0 \\ 1\end{array}\right], \quad B_{T}=\left[\begin{array}{ll}1 & 0 \\ 0 & p_{1}^{-1}\end{array}\right]$ $\left[\begin{array}{ll}B & 0\end{array}\right]$, and $J=\left[\begin{array}{ll}0 & 1 \\ 0 & 0\end{array}\right]$.

To get a compact form of (26):

$$
\left[\begin{array}{c}
\dot{x}_{T} \\
\dot{\Omega}_{T}
\end{array}\right]=\left[\begin{array}{cc}
A_{T}\left(\theta_{0}\right) & B_{T} \\
0 & J
\end{array}\right]\left[\begin{array}{c}
x_{T} \\
\Omega_{T}
\end{array}\right]
$$

Therefore, we considered a first local observer for $\Sigma_{T}$ :

$$
\begin{aligned}
{\left[\begin{array}{c}
\dot{\hat{x}}_{T} \\
\hat{\hat{\Omega}}_{T}
\end{array}\right] } & =\left[\begin{array}{cc}
A_{T}\left(\theta_{0}\right) & B_{T} \\
0 & J
\end{array}\right]\left[\begin{array}{c}
\hat{x}_{T} \\
\hat{\Omega}_{T}
\end{array}\right]+K_{T}\left(\theta_{0}\right)\left(y_{T}-\hat{y}_{T}\right), \\
\hat{y}_{T} & =C_{T} \hat{x}_{T}=\theta_{0},
\end{aligned}
$$

and the estimation error dynamic $e_{T}=\left[\begin{array}{l}x_{T} \\ \Omega_{T}\end{array}\right]-\left[\begin{array}{l}\hat{x}_{T} \\ \hat{\Omega}_{T}\end{array}\right]$ writes as:

$$
\dot{e}_{T}=\left(\left[\begin{array}{cc}
A_{T}\left(\theta_{0}\right) & B_{T} \\
0 & J
\end{array}\right]-K_{T}\left(\theta_{0}\right) C_{T}\right) e_{T},
$$

The design of $K_{T}\left(\theta_{0}\right)$ such that (41) is GAS is explained in section PI-Observers Cascade Design.

For the extended shoulder and the arm system $\Sigma_{A}$ (28), the observer can be derived paying attention that $\dot{\theta}_{0}$ and $\ddot{\theta}_{0}$ being non-measured, they are replaced with their estimate $\dot{\hat{\theta}}_{0}$ and $\ddot{\hat{\theta}}_{0}$. Thus, an expression is:

$$
\begin{gathered}
{\left[\begin{array}{cc}
E_{A}\left(\theta_{2}\right) & 0 \\
0 & I
\end{array}\right]\left[\begin{array}{c}
\dot{\hat{x}}_{A} \\
\dot{\hat{\Omega}}_{A}
\end{array}\right]=\left[\begin{array}{cc}
A_{A}\left(\hat{x}_{A}\right) & B_{A} \\
0 & J_{2}
\end{array}\right]\left[\begin{array}{c}
\hat{x}_{A} \\
\hat{\Omega}_{A}
\end{array}\right]+\left[\begin{array}{c}
0 \\
D_{a}\left(\theta_{A}\right) \\
0
\end{array}\right]} \\
+\left[\begin{array}{c}
0 \\
C\left(\theta_{A}\right) \\
0
\end{array}\right]\left[\begin{array}{l}
\dot{\hat{\theta}}_{0}^{2} \\
\dot{\hat{\theta}}_{0}
\end{array}\right]+K_{A}(\cdot)\left(y_{A}-\hat{y}_{A}\right) .
\end{gathered}
$$

Let us define the observation error $e_{A}=\left[\begin{array}{l}x_{A} \\ \Omega_{A}\end{array}\right]-\left[\begin{array}{l}\hat{x}_{A} \\ \Omega_{A}\end{array}\right]$ and writing $\left[\begin{array}{cc}E_{A}\left(\theta_{2}\right) & 0 \\ 0 & I\end{array}\right] \dot{e}_{A} \quad$ renders $\quad$ as: $\quad\left[\begin{array}{cc}E_{A}\left(\theta_{2}\right) & 0 \\ 0 & I\end{array}\right] \dot{e}_{A}=\left[\begin{array}{cc}A_{A}\left(x_{A}\right) & B_{A} \\ 0 & J_{2}\end{array}\right]\left[\begin{array}{c}x_{A} \\ \Omega_{A}\end{array}\right]$ $-\left[\begin{array}{cc}A_{A}\left(\hat{x}_{A}\right) & B_{A} \\ 0 & J_{2}\end{array}\right]\left[\begin{array}{c}\hat{x}_{A} \\ \hat{\Omega}_{A}\end{array}\right]-K_{A}(\cdot) C e_{A}+\left[\begin{array}{c}0 \\ C\left(\theta_{A}\right) \\ 0\end{array}\right]\left(\left[\begin{array}{c}\dot{\theta}_{0}^{2} \\ \ddot{\theta}_{0}\end{array}\right]-\left[\begin{array}{c}\hat{\theta}_{0}^{2} \\ \hat{\theta}_{0}\end{array}\right]\right)$

From where defining $\Delta A_{A}(\cdot)=A_{A}\left(x_{A}\right)-A_{A}\left(\hat{x}_{A}\right)$ and $\Delta \theta_{T}=$ $\left[\begin{array}{c}\dot{\theta}_{0}^{2} \\ \ddot{\theta}_{0}\end{array}\right]-\left[\begin{array}{c}\dot{\hat{\theta}}_{0}^{2} \\ \ddot{\hat{\theta}}_{0}\end{array}\right]$ as:

$$
\begin{gathered}
{\left[\begin{array}{cc}
E_{A}\left(\theta_{2}\right) & 0 \\
0 & I
\end{array}\right] \dot{e}_{A}=\left(\left[\begin{array}{cc}
A_{A}\left(x_{A}\right) & B_{A} \\
0 & J_{2}
\end{array}\right]-K_{A}(\cdot) C\right) e_{A}} \\
+\left[\begin{array}{c}
\Delta A_{A}(\cdot) \hat{x}_{A} \\
0
\end{array}\right]+\left[\begin{array}{c}
0 \\
C\left(\theta_{A}\right) \\
0
\end{array}\right]\left(\left[\begin{array}{c}
\dot{\theta}_{0}^{2} \\
\ddot{\theta}_{0}
\end{array}\right]-\left[\begin{array}{c}
\dot{\hat{\theta}}_{0}^{2} \\
\ddot{\hat{\theta}}_{0}
\end{array}\right]\right) .
\end{gathered}
$$

Now, in order to apply the result of Theorem 1, (43) must be adequately written as the second row of (32). The last part of (43) writes as: $\left[\begin{array}{c}\dot{\theta}_{0}^{2} \\ \dot{\theta}_{0}\end{array}\right]-\left[\begin{array}{c}\hat{\theta}_{0}^{2} \\ \hat{\theta}_{0}\end{array}\right]=\left[\begin{array}{cc}\dot{\theta}_{0}+\dot{\hat{\theta}}_{0} & 0 \\ 0 & 1\end{array}\right]\left[\begin{array}{l}\dot{\theta}_{0}-\dot{\hat{\theta}}_{0} \\ \ddot{\theta}_{0}-\hat{\hat{\theta}}_{0}\end{array}\right]$ from where:

$$
\begin{gathered}
{\left[\begin{array}{c}
0 \\
C\left(\theta_{A}\right) \\
0
\end{array}\right]\left(\left[\begin{array}{c}
\dot{\theta}_{0}^{2} \\
\ddot{\theta}_{0}
\end{array}\right]-\left[\begin{array}{c}
\dot{\hat{\theta}}_{0}^{2} \\
\ddot{\hat{\theta}}_{0}
\end{array}\right]\right)=\left[\begin{array}{c}
0 \\
C\left(\theta_{A}\right) \\
0
\end{array}\right]\left[\begin{array}{ccc}
\dot{\theta}_{0}+\dot{\hat{\theta}}_{0} & 0 & 0 \\
0 & 1 & 0
\end{array}\right]} \\
{\left[\begin{array}{c}
\dot{\theta}_{0}-\dot{\hat{\theta}}_{0} \\
\ddot{\theta}_{0}-\hat{\hat{\theta}}_{0} \\
\Omega_{T}-\hat{\Omega}_{T}
\end{array}\right]=-E_{12}\left(\theta_{A}, \dot{\theta}_{0}, \dot{\hat{\theta}}_{0}\right) \dot{e}_{T}}
\end{gathered}
$$

where $\quad E_{12}\left(\theta_{A}, \dot{\theta}_{0}, \dot{\hat{\theta}}_{0}\right)=-\left[\begin{array}{c}0 \\ C\left(\theta_{A}\right) \\ 0\end{array}\right]\left[\begin{array}{ccc}\dot{\theta}_{0}+\dot{\hat{\theta}}_{0} & 0 & 0 \\ 0 & 1 & 0\end{array}\right] . \quad$ The last transformation concerns the middle term of (43). Considering 


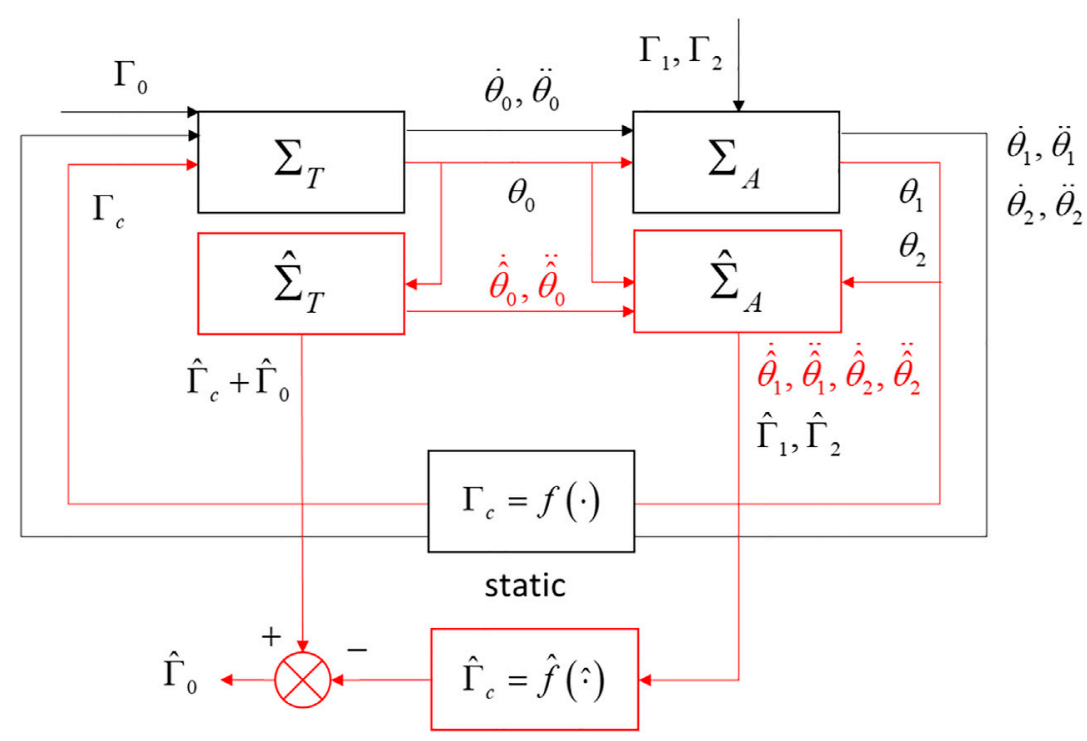

FIGURE 3 | 2-observers cascade for the decomposed S3S model.

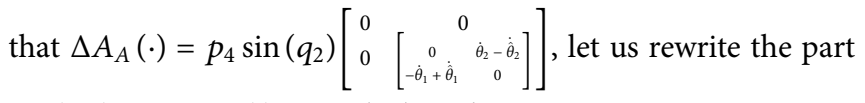
$A_{A}\left(x_{A}\right) e_{A}+\Delta A_{A}(\cdot) \hat{x}_{A}$ in (43) such as:

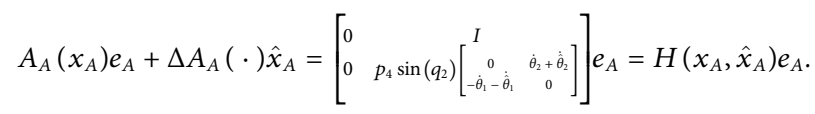

Finally, (43) can be transformed in the following:

$$
\begin{aligned}
& E_{12}\left(\theta_{A}, \dot{\theta}_{0}, \dot{\hat{\theta}}_{0}\right) \dot{e}_{T}+\left[\begin{array}{cc}
E_{A}\left(\theta_{2}\right) & 0 \\
0 & I
\end{array}\right] \dot{e}_{A} \\
& \quad=\left(\left[\begin{array}{cc}
H\left(x_{A}, \hat{x}_{A}\right) & B_{A} \\
0 & J_{2}
\end{array}\right]-K_{A}(\cdot) C\right) e_{A},
\end{aligned}
$$

and the full observation problem writes from (41) and (46) as:

$$
\begin{aligned}
& {\left[\begin{array}{c}
I \\
E_{12}(\cdot)
\end{array}\left[\begin{array}{cc}
E_{A}\left(\theta_{2}\right) & 0 \\
0 & I
\end{array}\right]\left[\begin{array}{c}
\dot{e}_{T} \\
\dot{e}_{A}
\end{array}\right]\right.}
\end{aligned}
$$

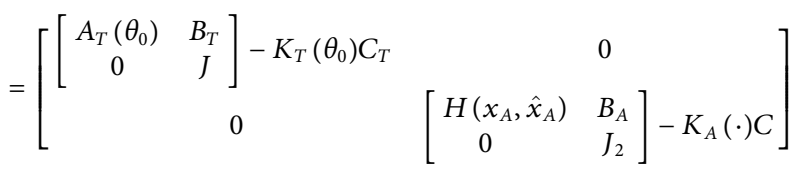

$$
\begin{aligned}
& \times\left[\begin{array}{l}
e_{T} \\
e_{A}
\end{array}\right] .
\end{aligned}
$$

Eq. (47) does correspond to the conditions of Theorem 1, the non-diagonal terms being bounded. Thus, conditions of Theorem one are fulfilled and the separation principle applies.

\section{PROPORTIONAL INTEGRAL-OBSERVERS CASCADE DESIGN}

\section{Proportional Integral-Observer for $\Sigma_{T}$}

The first observer (40) for the body trunk model $\Sigma_{T}$ is associated with the error dynamic model (41) recalled thereafter:

$$
\dot{e}_{T}=\left(\left[\begin{array}{cc}
A_{T}\left(\theta_{0}\right) & B_{T} \\
0 & J
\end{array}\right]-K_{T}\left(\theta_{0}\right) C_{T}\right) e_{T}
$$

As $A_{T}\left(\theta_{0}\right)=\left[\begin{array}{cc}0 & 1 \\ \beta p_{1}^{-1} p_{2} \frac{\sin \left(\theta_{0}\right)}{\theta_{0}} & 0\end{array}\right]$ and $C_{T}=\left[\begin{array}{llll}1 & 0 & 0 & 0\end{array}\right]$, consider the following observer gain: $K_{T}\left(\theta_{0}\right)=\left[\begin{array}{llll}0 & \beta p_{1}^{-1} p_{2} \frac{\sin \left(\theta_{0}\right)}{\theta_{0}} & 0 & 0\end{array}\right]^{T}+K_{L T}, \quad K_{L T} \in \mathbb{R}^{4 \times 1}$. Thus, the observation error is linear:

$$
\dot{e}_{T}=\left(\left[\begin{array}{cc}
J & B_{T} \\
0 & J
\end{array}\right]-K_{L T} C_{T}\right) e_{T}
$$

Any method can come at hand to determine $K_{L T}$. Therefore, the observer writes as:

$$
\left[\begin{array}{c}
\dot{\hat{x}}_{T} \\
\hat{\hat{\Omega}}_{T}
\end{array}\right]=\left[\begin{array}{cc}
A_{T}\left(\theta_{0}\right) & B_{T} \\
0 & J
\end{array}\right]\left[\begin{array}{c}
\hat{x}_{T} \\
\hat{\Omega}_{T}^{-}
\end{array}\right]-\left(\left[\begin{array}{llll}
0 & \beta p_{1}^{-1} p_{2} \frac{\sin \left(\theta_{0}\right)}{\theta_{0}} & 0 & 0
\end{array}\right]^{T}+K_{L T}\right)\left(y_{T}-\hat{y}_{T}\right) .
$$

\section{Proportional Integral-Observer for $\boldsymbol{\Sigma}_{\boldsymbol{A}}$}

The second observer (42) for the model $\Sigma_{A}$ is associated with a reduced error dynamic model issued from (46), with $H\left(x_{A}, \hat{x}_{A}\right)$ given in (45): 


$$
\left[\begin{array}{cc}
E_{A}\left(\theta_{2}\right) & 0 \\
0 & I
\end{array}\right] \dot{\varepsilon}_{A}=\left(\left[\begin{array}{cc}
H\left(x_{A}, \hat{x}_{A}\right) & B_{A} \\
0 & J_{2}
\end{array}\right]-K_{A}(\cdot) C\right) \varepsilon_{A} .
$$

In (51), it appears $\varepsilon_{A}$ as a slack variable different from $e_{A}$, as it is not the full-term error on the $\Sigma_{A}$ model. Thus, from the separation principle result if (41) and (51) are GAS, (47) is GAS.

The non-linear model (51) has a non-linear part $E_{A}\left(\theta_{2}\right)$ on the left. Of course as $E_{A}^{-1}\left(\theta_{2}\right)$ exists for every $\theta_{2}$ a classic representation of (51) could be used. Nevertheless, see Remark 1, a descriptor form is kept and the a polytopic representation of (51) is derived. $E_{A}\left(\theta_{2}\right)$ has one nonlinearity $\cos \left(q_{2}\right)$, and $H\left(x_{A}, \hat{x}_{A}\right)$ defined in (45) two non-linearities: $\sin \left(q_{2}\right)\left(\dot{\theta}_{1}+\dot{\hat{\theta}}_{1}\right)$ and $\sin \left(q_{2}\right)\left(\dot{\theta}_{2}+\dot{\hat{\theta}}_{2}\right)$. Therefore, a $2^{3}=8$ vertices corresponding to a polytopic model can be derived as:

$$
\begin{gathered}
\sum_{i=1}^{2} v_{i}\left(q_{2}\right)\left[\begin{array}{cc}
E_{A i} & 0 \\
0 & I
\end{array}\right] \dot{\varepsilon}_{A}=\sum_{i=1}^{2} \sum_{j=1}^{2} w_{1 i}\left(x_{A}, \hat{x}_{A}\right) w_{2 j}\left(x_{A}, \hat{x}_{A}\right) \\
\times\left(\left[\begin{array}{cc}
H_{i j} & B_{A} \\
0 & J_{2}
\end{array}\right]-K_{A}(\cdot) C\right) \varepsilon_{A},
\end{gathered}
$$

with $\quad \cos \left(q_{2}\right) \in[v, 1], \quad v_{1}\left(q_{2}\right)=\frac{1-\cos \left(q_{2}\right)}{1-v}=1-v_{2}\left(q_{2}\right)$, $\sin \left(q_{2}\right)\left(\dot{\theta}_{i}+\dot{\hat{\theta}}_{i}\right) \in\left[\rho_{i}, \bar{\rho}_{i}\right], \quad i \in\{1,2\} \quad w_{i 1}\left(x_{A}, \hat{x}_{A}\right)=$ $\frac{\bar{\rho}_{i}-\sin \left(q_{2}\right)\left(\dot{\theta}_{i}+\hat{\theta}_{i}\right)}{\bar{\rho}_{i}-\underline{\rho}_{i}}=1-w_{i 2}\left(x_{A}, \hat{x}_{A}\right), i \in\{1,2\}$ and the matrices:

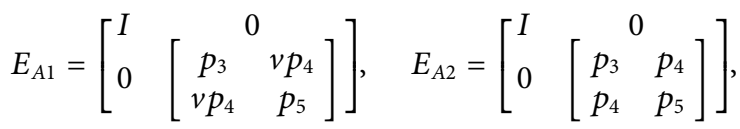

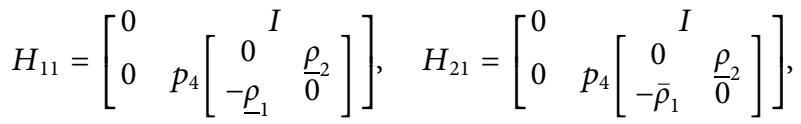

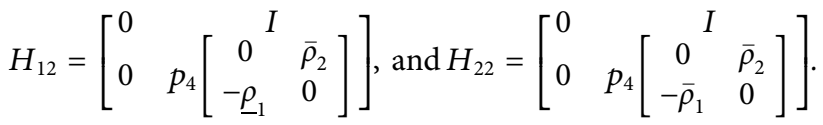

From this polytopic description, as usual, an extended description of (52) is used (Taniguchi et al., 2000). The extended state $\varepsilon^{*}=\left[\begin{array}{c}\varepsilon_{A} \\ \dot{\varepsilon}_{A}\end{array}\right]$ is chosen together using $\bar{E}=\left[\begin{array}{ll}I & 0 \\ 0 & 0\end{array}\right]$ and with a Lyapunov function:

$$
V\left(\varepsilon^{*}\right)=\varepsilon^{* T} \bar{E}^{T} P \varepsilon^{*}, \quad P=\left[\begin{array}{cc}
P_{1} & 0 \\
P_{3} & P_{4}
\end{array}\right], \quad P_{1}=P_{1}^{T}>0 .
$$

The observer gain can only depend on measured variables; therefore, due to the definitions of $w_{i 1} i, j \in\{1,2\}$, only $v_{i}\left(q_{2}\right)$ is concerned:

$$
\bar{E} \dot{\varepsilon}^{*}=\left(\bar{A}_{v w_{1} w_{2}}-P^{-T} \bar{K}_{v} \bar{C}\right) \mathcal{E}^{*},
$$

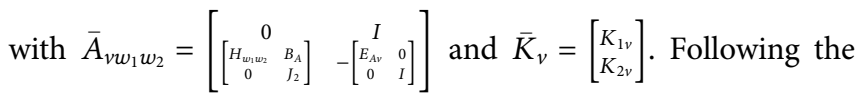
study of Guerra et al. (2015), a LMI constraint problem is given by the following:
Find $P=\left[\begin{array}{cc}P_{1} & 0 \\ P_{3} & P_{4}\end{array}\right], \quad P_{1}=P_{1}^{T}>0,\left[\begin{array}{l}K_{1 i} \\ K_{2 i}\end{array}\right]$ such that, $i, j, k \in\{1,2\}$

$$
\left[\begin{array}{c}
P_{3}^{T}\left[\begin{array}{cc}
H_{j k} & B_{A} \\
0 & J_{2}
\end{array}\right]-K_{1 i} C+(*) \\
P_{4}^{T}\left[\begin{array}{cc}
H_{j k} & B_{A} \\
0 & J_{2}
\end{array}\right]-K_{2 i} C+P_{1}-\left[\begin{array}{cc}
E_{A i} & 0 \\
0 & I
\end{array}\right]^{T} P_{3}-P_{4}^{T}\left[\begin{array}{cc}
E_{A i} & 0 \\
0 & I
\end{array}\right]-\left[\begin{array}{cc}
E_{A i} & 0 \\
0 & I
\end{array}\right]^{T} P_{4}
\end{array}\right]<0 .
$$

At the end, the observer for $\Sigma_{A}$ is, with $P=\left[\begin{array}{cc}P_{1} & 0 \\ P_{3} & P_{4}\end{array}\right], \quad P_{1}=P_{1}^{T}>0,\left[\begin{array}{c}K_{1 i} \\ K_{2 i}\end{array}\right] i \in\{1,2\}$, solution of, (56):

$$
\begin{aligned}
\sum_{i=1}^{2} v_{i}\left(q_{2}\right)\left[\begin{array}{cc}
E_{A i} & 0 \\
0 & I
\end{array}\right]\left[\begin{array}{c}
\dot{\hat{x}}_{A} \\
\dot{\hat{\Omega}}_{A}
\end{array}\right]=\left[\begin{array}{cc}
A_{A}\left(\hat{x}_{A}\right) & B_{A} \\
0 & J_{2}
\end{array}\right]\left[\begin{array}{c}
\hat{x}_{A} \\
\hat{\Omega}_{A}
\end{array}\right] \\
+\left[\begin{array}{c}
0 \\
D_{a}\left(\theta_{A}\right) \\
0
\end{array}\right]+\left[\begin{array}{c}
0 \\
C\left(\theta_{A}\right) \\
0
\end{array}\right]\left[\begin{array}{c}
\dot{\hat{\theta}}_{0}^{2} \\
\ddot{\hat{\theta}}_{0}
\end{array}\right]+\left[\sum_{i=1}^{2} v_{i}\left(q_{2}\right)\left[\begin{array}{cc}
E_{A i} & 0 \\
0 & I
\end{array}\right] I\right] P_{(\cdot)}^{-T} \\
\times \sum_{i=1}^{2} v_{i}\left(q_{2}\right)\left[\begin{array}{c}
K_{1 i}(\cdot) \\
K_{2 i}(\cdot)
\end{array}\right]\left(y_{A}-\hat{y}_{A}\right) .
\end{aligned}
$$

At last for the coupling term $\Gamma_{c}$ (25):

$$
\begin{aligned}
& \hat{\Gamma}_{c}=\left[\begin{array}{llll}
-p_{10} \sin \left(q_{1}\right) \dot{\hat{\theta}}_{1} & -p_{9} \sin \left(q_{1}+q_{2}\right) \dot{\hat{\theta}}_{2} & p_{10} \cos \left(q_{1}\right) & p_{9} \cos \left(q_{1}+q_{2}\right)
\end{array}\right] \\
& \times\left[\begin{array}{c}
\dot{\hat{x}}_{A} \\
\dot{\hat{\Omega}}_{A}
\end{array}\right]-\hat{\Gamma}_{1} \text {. }
\end{aligned}
$$

\section{Linear Matrix Inequalities Solutions}

For the global continuous PI-observer, solving the LMI constraints problem (18) in the compact set $\Omega_{x}(\mathbf{1 7})$ is feasible. To enhance the performances, a decay rate (add $\tau P_{1}, \tau>0$ in the first entry of constraints (18)) and a norm limitation on the gains $\left[\begin{array}{ll}K_{1 i} & K_{2 i}\end{array}\right]\left[\begin{array}{l}K_{1 i} \\ K_{2 i}\end{array}\right] \leq \varepsilon I_{3}$ are used. The second part, using a Schur's

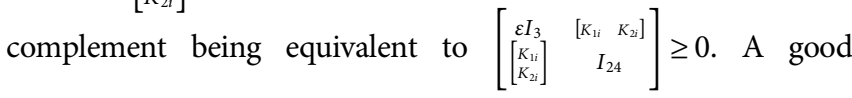
compromise was obtained using a decay rate of $\tau=6$ and $\varepsilon=10^{6}$. It is useless to present the full set of matrices solution of the problem, just the extreme of the polytope of the gains $K_{2,1}$ and $K_{2,16}$ are given to exemplify:

$$
K_{2,1}=\left[\begin{array}{ccc}
71.1 & 4.3 & -2.2 \\
-0.5 & 111 & -33 \\
-2.9 & -35.3 & 222 \\
79 & 12 & -2 \\
10.5 & 45.4 & 12 \\
1.7 & 13.4 & 1.1 \\
-1.6 & 1.4 & 0.01 \\
-0.33 & -12.3 & -6 \\
1.9 & -4.7 & 8.5 \\
0 & 0 & 0 \\
0 & 0 & 0 \\
0 & 0 & 0
\end{array}\right], K_{2,16}=\left[\begin{array}{ccc}
66.2 & -0.7 & -1.8 \\
-2.3 & 115 & -36 \\
-4.3 & -35 & 230 \\
83 & 7.5 & 3.8 \\
7.4 & 50 & 17.6 \\
2.8 & 16.8 & -2.2 \\
-1.5 & -1.6 & -0.5 \\
-7.7 & -5.7 & -9.5 \\
7.8 & -7 & 23 \\
0 & 0 & 0 \\
0 & 0 & 0 \\
0 & 0 & 0
\end{array}\right] .
$$

Local trunk observer gain $K_{L T}$ is easily calculated via pole placement, after some trials-and-errors. The solution 

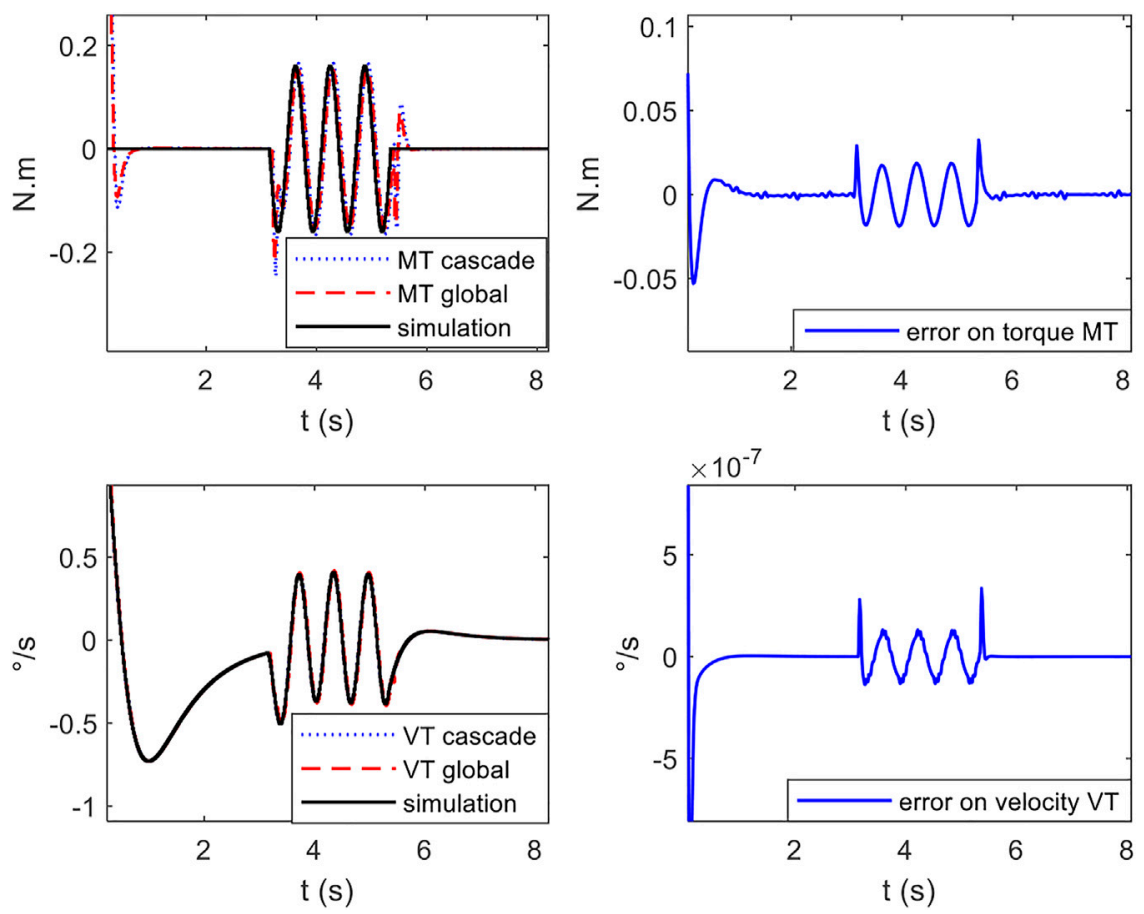

FIGURE 4 | left: estimation of lumbar velocity and torque by cascade observer and global observer compared to simulation; right: estimation error.
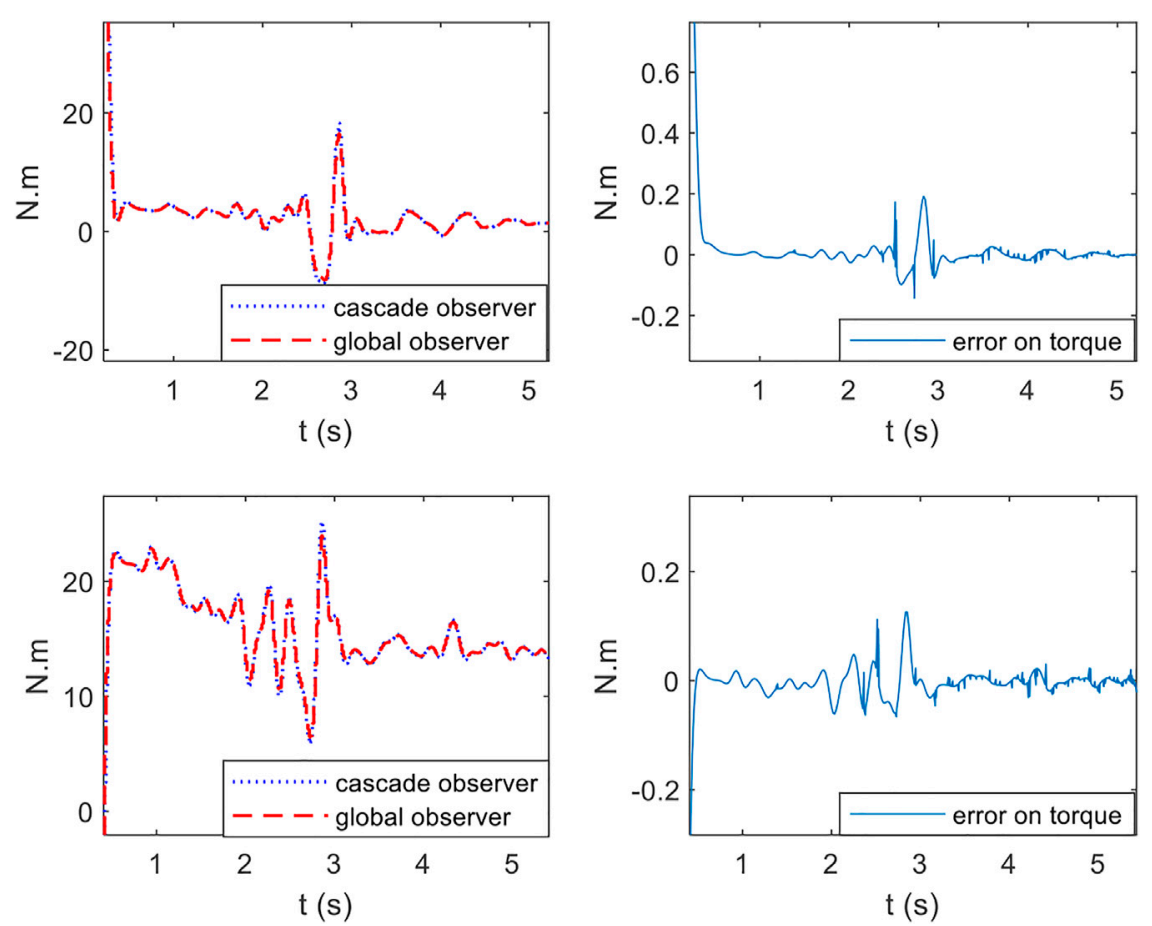

FIGURE 5 | real-time lumbar torque estimated by cascade observer and global observer: top first subject and bottom second subject. 
of (49) for the model $\Sigma_{T}$ observer is: $K_{L T}=10^{2}\left[\begin{array}{llll}6 & 4 & 5.4 \times 10^{2} & 1.9 \times 10^{2}\end{array}\right]^{T}$.

For the problem (56), a decay rate of $\tau=7$ has been added in the first entry of (56), and the solution adopted by solving LMIs problem is $\left[\begin{array}{l}K_{1 i}(\cdot) \\ K_{2 i}(\cdot)\end{array}\right], i \in\{1,2\}$ where:

$$
\begin{aligned}
K_{11} & =\left[\begin{array}{rr}
141.2 & -31.3 \\
-31.3 & 144.4 \\
11.8 & -3 \\
-3.6 & 12.7 \\
8 \times 10^{-3} & 6 \times 10^{-3} \\
3 \times 10^{-3} & -5 \times 10^{-3} \\
10^{-3} & 2 \times 10^{-3} \\
10^{-4} & -3 \times 10^{-3}
\end{array}\right], K_{12}=\left[\begin{array}{cc}
131.6 & -27.1 \\
-21.2 & 122.6 \\
10.7 & -2.7 \\
-1.4 & 9.8 \times 10^{-1} \\
2 \times 10^{-3} & 4 \times 10^{-3} \\
8 \times 10^{-3} & -3.3 \times 10^{-1} \\
10^{-3} & 9 \times 10^{-3} \\
10^{-4} & -21 \times 10^{-4}
\end{array}\right], \\
-4.6 & -4.5 \\
K_{21} & =\left[\begin{array}{rr}
15.3 & -7.5 \\
-2.7 \times 10^{-1} & 92 \times 10^{-2} \\
-3.6 \times 10^{-2} & -18 \times 10^{-2} \\
6 \times 10^{-4} & 49 \times 10^{-3} \\
13 \times 10^{-4} & 8 \times 10^{-3} \\
10^{-4} & 2 \times 10^{-3} \\
10^{-3} & -3 \times 10^{-4}
\end{array}\right], K_{22}=\left[\begin{array}{rr}
-4.98 \\
-2 \times 10^{-1} & 8.7 \times 10^{-1} \\
-2 \times 10^{-2} & -5 \times 10^{-2} \\
8 \times 10^{-4} & 5 \times 10^{-3} \\
5 \times 10^{-3} & 6 \times 10^{-3} \\
10^{-4} & 2.7 \times 10^{-3} \\
10^{-3} & -1.6 \times 10^{-2}
\end{array}\right] .
\end{aligned}
$$

\section{VALIDATION AND EXPERIMENTAL RESULTS}

\section{Validation in Simulation}

Simulations were run using MATLAB software R2019b and YALMIP interface on a computer with a $2.6 \mathrm{GHZ}$ processor. A MOSEK solver is chosen as the numeric calculating tool to solve LMI problems.

A full non-linear S3S controlled model in a closed-loop is simulated and acts like a black box with the angles as outputs. Between 2 and $3 \mathrm{~s}$ a sinusoidal disturbance is added on the lumbar velocity, it corresponds to the amplitude of the accelerations used during rehabilitation exercises in living subjects with SCI (Bjerkefors et al., 2007). For simulation purposes, a passive lumbar contribution defined as a sinusoidal signal with an amplitude of $0.15 \mathrm{~N} . \mathrm{m}$ and frequency $2 \mathrm{Hzis}$ added as an input to the nonlinear model. An example of simulation gives the estimated lumbar couple and velocity of the global 2D-S3S observer and the cascade local observer under the same constraints.

Figure 4 shows one result. Only the speed $\dot{q}_{0}$ and the torque $T_{0}$ at the trunk are shown as they present the farthest variable from the measured angles, thus the ones that cumulate all the errors. Both observers exhibit similar performances and errors (less than $5 \%$ on the torque), showing that they are suitable to solve the problem.

\section{Real-Time Experiments}

The protocol in real-time experiments is similar to simulation context with human joint angles resulting from experimental manipulations. The experiments were carried out according to the agreement of "comité d'éthique pour la recherche du Centre de Recherche Interdisciplinaire en réhabilitation du Grand-Montréal (CRIR-1083-0515R)." Two subjects were treated with different profiles: a 32-year-old woman (weight: $55 \mathrm{~kg}$, tall: $162 \mathrm{~cm}$ ) having a SCI in vertebra T6 for 3 years and a 53 -year-old man (weight: $100 \mathrm{~kg}$, tall: $180 \mathrm{~cm}$ ) suffering from a SCI in vertebra in T11 for 10 years.

The experimental protocol is as follows: the subject is asked to keep his seated balance while applying a disturbing force to the level of the T6 vertebra of the trunk. Once disturbed, each subject tries to stabilize himself by designing a compensatory strategy using the upper limbs. Figure 5 gives the estimated real-time lumbar couple of the global 2D-S3S observer and the cascade local observer as well as the difference. Results of the real-time experiments confirm the simulation results and show the similarity between the response of the global observer and the cascade observer. The difference in behaviors after applying the disturbing force depends on the level of the injury and its severity. Each subject in order to recover a stable behavior, has his/her own stabilization strategy based on the upper body part.

At last, note that the Trunk torque is positive at the beginning of each experimental acquisition, which makes sense from a mechanical balance point of view because the angle $q_{0}$ is negative at that time (a positive articular torque rotates the segment counterclockwise). Once the disturbing force applied, the joint torque decreases. This significant reduction is related to the disturbance which pushes each subject in the direction anterior to what is equivalent to injecting a negative torque (resp. positive) at the level of the trunk (resp. of the shoulder) (Blandeau, 2018).

\section{CONCLUSION}

People living with a SCI sitting position has been described via a so-called S3S model (Blandeau, 2018). The main goal is to be able to understand the different strategies that can be used by the people with the SCI. The internal control to stabilize the 2DS3S model was out of the scope of this study and previously solved in Guerra et al. (2020). Understanding the strategies amounts to finding the torques that are unmeasured variables. A first PI-observer was derived in a discrete form in Blandeau (2018). In continuous, due to the problem of unmeasured variables in the premises of the quasi-LPV model, the problem was not solved. The first part of this study answered to this question especially thinking to push farther as possible the building of the polytope. The next step is to go to a more precise model especially including the sagittal plane, thus going to a 2D-S3S to its $3 \mathrm{D}-\mathrm{S} 3 \mathrm{~S}$ form. Even if perfectly suitable for the 2D-S3S model, the global PI-observer solution ends with number of variables and LMI constraints that is very certainly not compatible for the 3D-S3S with actual solvers.

Therefore, two different solutions can apply. The first one consists in simplifying the model (partial linearization for example) but then introduces modeling errors in the observer. 
The second one, presented in this study, consists in using a cascade of observers. From an initial separation-like property, the 2D-S3S has been decomposed via two descriptors quasi-LPV observers, which design involved much more simplified LMI constraints problems.

Simulation and real-time experiments show that both approaches are suitable for the 2D-S3S model. Thus, not only the continuous global PI-observer design is validated but also the cascade observers using a decomposed form. Thus, it also validates the future steps to get a solution for a 3D-S3S model.

Thereby, as a final goal, we will be able to provide the occupational therapist with real time torques during rehabilitation exercises in order to follow the learning of new stabilization strategies outside the sagittal plan.

\section{DATA AVAILABILITY STATEMENT}

The original contributions presented in the study are included in the article/Supplementary Material; further inquiries can be directed to the corresponding author.

\section{ETHICS STATEMENT}

The studies involving human participants were reviewed and approved by comité d'éthique pour la recherche du Centre de

\section{REFERENCES}

Blandeau, M., Estrada-Manzo, V., Guerra, T. M., Pudlo, P., and Gabrielli, F. (2018). Fuzzy unknown input observer for understanding sitting control of persons living with spinal cord injury. Eng. Appl. Artif. Intelligence 67, 381-389. doi:10.1016/j.engappai.2017.09.016

Blandeau, M. (2018). Modélisation Et Caractérisation De La Stabilité En Position Assise Chez Les Personnes Vivant Avec Une Lésion De La Moelle Épinière. PhD Thesis. Université de Valenciennes et du Hainaut-Cambresis. Valenciennes: Université Polytechnique Hauts-de-France.

Bouarar, T., Guelton, K., and Manamanni, N. (2010). Robust fuzzy Lyapunov stabilization for uncertain and disturbed Takagi-Sugeno descriptors. ISA Trans. 49 (4), 447-461. doi:10.1016/j.isatra.2010.06.003

Boyd, S., Ghaoui, L. E., Feron, E., and Balakrishnan, V. (1994). Linear Matrix Inequalities in System and Control Theory. Pennsylvania: Society for Industrial and Applied Mathematics.

Chadli, M., and Darouach, M. (2012). Novel bounded real lemma for discrete-time descriptor systems: Application to Ho control design. Automatica 48 (2), 449-453. doi:10.1016/j.automatica.2011.10.003

Chen, J., Patton, R. J., and Zhang, H.-Y. (1996). Design of unknown input observers and robust fault detection filters. Int. J. Control 63 (1), 85-105. doi:10.1080/ 00207179608921833

Fang, Y., Morse, L. R., Nguyen, N., Tsantes, N. G., and Troy, K. L. (2017). Anthropometric and biomechanical characteristics of body segments in persons with spinal cord injury. J. Biomech. 55, 11-17. doi:10.1016/j.jbiomech.2017.01.036

Grip, H. F., Saberi, A., and Johansen, T. A. (2012). Observers for interconnected nonlinear and linear systems. Automatica 48 (7), 1339-1346. doi:10.1016/ j.automatica.2012.04.008

Guerra, T.-M., Bernal, M., and Blandeau, M. (2018). Reducing the Number of Vertices in Some Takagi-Sugeno Models: Example in the Mechanical Field. IFAC-PapersOnLine 51 (10), 133-138. doi:10.1016/ j.ifacol.2018.06.250
Recherche Interdisciplinaire en réhabilitation du GrandMontréal (CRIR-1083-0515R). The patients/participants provided their written informed consent to participate in this study.

\section{AUTHOR CONTRIBUTIONS}

All authors listed have made a substantial, direct, and intellectual contribution to the work and approved it for publication.

\section{FUNDING}

This work was partly supported by Zodiac Seats France and Direction Générale de l'Aviation Civile (project n²014 930181). The present research work has been supported by the European Community, the French Ministry de l'Education Nationale, de la Recherche et de la Technologie, the Region Hauts-de-France, the Centre National de la Recherche Scientifique (CNRS); in part through the project ELSAT2020.

\section{ACKNOWLEDGMENTS}

The authors gratefully acknowledge the support of these institutions.

Guerra, T. M., Blandeau, M., Nguyen, A. T., Srihi, H., and Dequidt, A. (2020). "Stabilizing unstable biomechanical model to understand sitting stability for persons with spinal cord injury," in IFAC- PapersOnLine (Berlin, Gemany: World Congress). doi:10.1016/j.ifacol.2020.12.2225

Guerra, T. M., Estrada-Manzo, V., and Lendek, Z. (2015). Observer design for Takagi-Sugeno descriptor models: An LMI approach. Automatica 52, 154-159. doi:10.1016/j.automatica.2014.11.008

Ichalal, D., and Guerra, T. M. (2019). Decoupling unknown input observer for nonlinear quasi-LPV systems. IEEE 58th Conf. Decis. Control. (Cdc), 3799-3804. doi:10.1109/cdc40024.2019.9029339

Khalil, W., and Dombre, E. (2004). Google-Books-ID: nyrYoPu5kloC. Modeling, Identification and Control of Robots. UK: Butterworth-Heinemann.

Lendek, Z., Nagy, Z., and Lauber, J. (2018). Local stabilization of discrete-time TS descriptor systems. Eng. Appl. Artif. Intelligence 67, 409-418. doi:10.1016/ j.engappai.2017.09.006

Lendek, Zs., Babuska, R., and De Schutter, B. (2008). Stability of cascaded fuzzy systems and observers. IEEE Trans. Fuzzy Syst. 17 (3), 641-653.

Lendek, Zs., Guerra, T. M., Babuska, R., and De Schutter, B. (2010). Stability Analysis and nonlinear observer design using Takagi-Sugeno fuzzy models. Stud. fuzziness soft Comput. Vol. 262, 2010938248, 2010 . Library of Congress Control Number.

Potten, Y. J. M., Seelen, H. A. M., Drukker, J., Reulen, J. P. H., and Drost, M. R. (1999). Postural muscle responses in the spinal cord injured persons during forward reaching. Ergonomics 42, 1200-1215. doi:10.1080/001401399185081

Skelton, R. E., Iwasaki, T., and Grigoriadis, D. E. (1997). A Unified Algebraic Approach to Control Design. Routledge, 1997.

Tanaka, K., and Wang, O. H. (2001). Fuzzy Control Systems Design and Analysis: A Linear Matrix Inequality Approach. New York: Wiley.

Taniguchi, T., Tanaka, K., and Wang, H. O. (2000). Fuzzy descriptor systems and nonlinear model following control. IEEE Trans. Fuzzy Syst. 8 (4), 442-452. doi:10.1109/91.868950

Varga, A. (1995). On stabilization methods of descriptor systems. Syst. Control. Lett., 1995. doi:10.1016/0167-6911(94)00017-p 
Zemouche, A., Boutayeb, M., and Bara, G. I. (2008). Observers for a class of Lipschitz systems with extension to performance analysis. Syst. Control. Lett. 57 (1), 18-27. doi:10.1016/j.sysconle.2007.06.012

Conflict of Interest: The authors declare that the research was conducted in the absence of any commercial or financial relationships that could be construed as a potential conflict of interest.

Publisher's Note: All claims expressed in this article are solely those of the authors and do not necessarily represent those of their affiliated organizations, or those of the publisher, the editors, and the reviewers. Any product that may be evaluated in this article, or claim that may be made by its manufacturer, is not guaranteed or endorsed by the publisher.

Copyright (C) 2021 Srihi, Guerra, Nguyen, Pudlo and Dequidt. This is an open-access article distributed under the terms of the Creative Commons Attribution License (CC $B Y)$. The use, distribution or reproduction in other forums is permitted, provided the original author(s) and the copyright owner(s) are credited and that the original publication in this journal is cited, in accordance with accepted academic practice. No use, distribution or reproduction is permitted which does not comply with these terms. 